\title{
Grundlagen zur Sprachbildung in der Lehramtsausbildung Mathematik
}

\author{
Inhaltsverzeichnis
}

1.1 Einleitung: Sprachbildung in der Hochschullehre allgemein und fachbezogen - 4

1.1.1 Die Beziehung von fach- und sprachdidaktischen Konzeptbildungen für die Hochschullehre - 8

1.1.2 Der Zusammenhang von Denken und Sprechen im Studium - 16

1.1.3 Zur Bedeutung von Sprache für das Fach Mathematik - 20

1.2 Leitideen und Design-Prinzipien für eine sprachbewusste Hochschullehre in der Lehramtsausbildung Mathematik - 23

1.2.1 Leitideen zur Verortung von Sprache und Fach in der Lehramtsausbildung Mathematik - 24

1.2.2 Hochschuldidaktische Design-Prinzipien für eine sprachbewusste Lehramtsausbildung Mathematik - 41

1.2.3 Leitideen und Design-Prinzipien im Überblick - 47

1.3 Lehramtsausbildung interdisziplinär - 48

1.3.1 Curriculare Verortung - 48

1.3.2 Seminarkonzeption - 50 
Sprache gilt als das zentrale Medium fachlichen Lernens in Bildungseinrichtungen (Becker-Mrotzek et al. 2013; Schmölzer-Eibinger und Thürmann 2015). Ausreichende Kompetenzen in der Sprache, in der der Unterricht stattfindet - und das bedeutet für viele Lernende: in ihrer Zweitsprache ${ }^{1}$ - sind die Voraussetzung dafür, am Bildungsprozess erfolgreich teilhaben zu können. Dass dies auch für vermeintlich „sprachferne“ Fächer wie Mathematik gilt, belegt die beeindruckende Fülle von Forschungsbefunden und Entwicklungsarbeiten zur Sprachbildung im Mathematikunterricht.

Der Themenkomplex ist gerade mit Blick auf die mathematikdidaktischen Arbeiten ein wichtiges Forschungs- und Entwicklungsfeld, das in den letzten Jahren zunehmend an Bedeutung gewonnen hat. So ist es ein großes Verdienst grundlegender Arbeiten, etwa Interaktionsmuster und Routinen im Mathematikunterricht herauszuarbeiten (vgl. z. B. Voigt 1984, 1994; Krummheuer und Voigt 1991), die sprachlichen Eigenschaften des Fachs Mathematik detailliert zu erfassen (vgl. z. B. Maier und Schweiger 1999) oder Detailanalysen von Kommunikation im Mathematikunterricht vorzunehmen (vgl. Pimm 1987; von Kügelgen 1994).

In den Fokus einer breiteren öffentlichen Diskussion rückte das Thema insbesondere durch die großen Schulleistungsstudien (z. B. Secada 1992; Stanat 2006; OECD 2007), die u. a. Disparitäten der Mathematikleistungen zwischen lebensweltlich ein- und mehrsprachig aufwachsenden Schüler*innen zu Tage förderten. In diesem Zusammenhang konnte insbesondere gezeigt werden, dass nicht Mehrsprachigkeit an sich der entscheidende Faktor für entsprechende Leistungsdisparitäten ist, sondern vielmehr die Sprachkompetenz in der Unterrichtssprache Deutsch als Medium der fachbezogenen Verständigung (vgl. Prediger et al. 2015, Prediger und Redder 2020). Weiterhin belegen empirische Studien die besondere Bedeutung von sprachlicher Kompetenz für das Mathematiklernen (Heinze et al. 2009; Prediger 2010, Prediger et al. 2018). Dass Sprachbildung als Aufgabe aller Fächer mittlerweile eine ganz selbstverständliche - und auch curricular festgeschriebene - Forderung ist, lässt sich mithin auch auf die entsprechenden Konsequenzen zurückführen, die im Umfeld und im Nachgang der breit angelegten Schulleistungsstudien gezogen wurden.

Auch methodisch wird Sprache im Mathematikunterricht durchaus sehr breit beforscht: So finden sich theoretische Arbeiten etwa zur Vielfalt von Forschungsrahmen in diesem Themenfeld (z. B. Planas und Schütte 2018), eher quantitativ orientierte Studien (z. B. Leiss et al. 2019; Prediger et al. 2015; Ufer et al. 2013) oder qualitativrekonstruktive Arbeiten (z. B. Götze 2019).

Für den Kontext einer sprachbewussten Hochschullehre in der Lehramtsausbildung Mathematik sind dabei gerade solche Arbeiten von Bedeutung, die der Frage nachgehen, wie der Kluft zwischen empirischen Befunden und der unterrichtlichen Realität zum Thema Sprachbildung im Fach begegnet werden kann. Die Entwicklung und Beforschung entsprechender Lerndesigns wird etwa im Rahmen von fachdidaktischen Entwicklungsforschungsprojekten realisiert. Hier haben sich in den letzten Jahren ganz unterschiedli-

1 Der Begriff „Zweitsprache“ wird hier verwendet, auch wenn z. B. in Deutschland das Deutsche für viele (insbes. auch neuzugewanderte) Schüler*innen ihre Dritt- oder Viertsprache ist. Im englischsprachigen Raum wird anstelle von „Zweitsprache“ - ,second language“ - daher zunehmend der Ausdruck ,,additional language" verwendet (also: EAL - English as an Additional Language / Englisch als zusätzliche Sprache). Eine Entsprechung (,Deutsch als zusätzliche Sprache“) hat sich im deutschsprachigen Raum bislang noch nicht etabliert. 
che Ansätze herausgebildet, deren gemeinsames Kennzeichen die Entwicklung von Lerndesigns im Rahmen iterativer Forschungs- und Entwicklungszyklen ist: Design Science (Wittmann 1995), Design-Based Research (Barab und Squire 2004; Brown 1992; Collins 1992), Developmental Research (Freudenthal 1991; Gravemeijer 1994; Gravemeijer und Cobb 2006), Engineering Research (Burkhardt 2006). Entwicklungsforschungsprojekte adressieren mithin ganz bewusst die Frage nach dem konstruktiven Umgang (für den vorliegenden Kontext) mit Sprachbildung im Mathematikunterricht: So beschreibt etwa Wessel (2015) im Rahmen von Designexperimenten ein Förderkonzept zum Thema Sprachbildung im Mathematikunterricht am Beispiel von „Anteilen“ und arbeitet spezifische kommunikationsfördernde Designprinzipien, den Zusammenhang von sprachlichen Registern und der Verwendung bzw. Vernetzung unterschiedlicher Darstellungen sowie die verstehensförderliche Wirkung gezielt eingesetzter Sprachmittel im Sinne eines Scaffolding heraus. Der Ansatz der Entwicklungsforschung wird auch im Kontext der hier vorgestellten Konzepte für eine sprachbewusste Hochschullehre genauer beleuchtet mit Blick auf die Frage, inwiefern er sich als Gestaltungselement im Studium als produktiv erweist.

Insgesamt belegen die wissenschaftlichen Befunde zum Thema Sprachbildung im Mathematikunterricht die Bedeutung der durchgängigen und nachhaltigen Vernetzung von sprachlichem und fachlichem Lernen, um für alle Lernenden den bestmöglichen Zugang zu Bildung zu gewährleisten - auch (aber nicht nur) angesichts des hohen Anteils an mehrsprachigen Schüler*innen. Eine entsprechende Qualifizierung der zukünftigen Lehrkräftegeneration für Sprachbildung im Fach ist daher ein dringendes gesellschaftliches und bildungspolitisches Desiderat.

Im ersten Kapitel werden zunächst Rahmenbedingungen und Grundlagen für eine universitäre Lehramtsausbildung Mathematik vorgestellt, in der Fragen fachlichen und sprachlichen Lernens systematisch aufeinander bezogen werden. Wir sprechen in diesem Zusammenhang kurz von sprachbewusster Hochschullehre. In Abschn. 1.1 wird die Notwendigkeit einer fachspezifischen Vorbereitung von Studierenden auf die Umsetzung von Sprachbildung im Unterricht begründet. Anhand dreier Beispiele wird aufgezeigt, wo sich auf Hochschulebene günstige Schnittstellen für die Vernetzung von Mathematik (-didaktik) und Sprachbildung ergeben, und zwar (1) in Bezug auf die Verknüpfung fachund sprachdidaktischer Konzeptbildungen, (2) mit Blick auf Zusammenhänge von Denken und Sprechen im Studium sowie (3) hinsichtlich der Bedeutung von Sprache für das Fach Mathematik. Das sehr reichhaltig bearbeitete und nach wie vor aktuelle Forschungsfeld „Mathematik und Sprache“ wird dabei bewusst nur exemplarisch diskutiert, sodass der eigentliche Schwerpunkt - nämlich die Ausarbeitung von Konzepten für eine sprachbewusste Hochschullehre - in den Blick genommen werden kann; auf die einschlägigen Forschungsarbeiten wird an den entsprechenden Stellen verwiesen.

Dies gilt insbesondere auch für den $>$ Abschn. 1.2, der die theoretischen Orientierungen für das Buch darlegt: In $>$ Abschn. 1.2.1 werden zunächst Leitideen zur Verortung von Sprache und Fach in der Lehramtsausbildung Mathematik formuliert, an denen sich die Inhalte der Lehrveranstaltungen orientieren. In $>$ Abschn. 1.2.2 werden hochschuldidaktische Design-Prinzipien begründet, die für die praktische Gestaltung der Hochschullehre zentral sind. Abschließend wird in Abschn. 1.3 ein Seminarkonzept konkretisiert und curricular verortet, das der beschriebenen Konzeption für eine sprachbewusste Hochschullehre zugrunde liegt. 


\subsection{Einleitung: Sprachbildung in der Hochschullehre - allgemein und fachbezogen}

I) Ich glaube nicht, dass es irgendeinen Mathematikunterricht gibt, in dem man keine sprachförderlichen Elemente braucht (Studierender, Praxissemester 2018).

Sprache und Fach sind auf das Engste miteinander verwoben. Sprache ist nicht nur Voraussetzung und Medium fachlichen Lernens, sondern auch selbst Lerngegenstand (Leisen 2010 ff., Becker-Mrotzek et al. 2013, Prediger 2013). So konstatieren etwa BeckerMrotzek et al. (2013):

॥I In den letzten Jahren hat sich zunehmend herausgestellt, wie sehr Sprache (und nicht nur Fachsprache) konstitutiv ist für das Lehren und Lernen in allen schulischen Fächern, von den Gesellschaftswissenschaften über Naturwissenschaften bis hin zur Mathematik. Es geht dabei konkret um den Aufbau von fachbezogenen Verstehens- und Mitteilungsfähigkeiten, die sich offenbar nicht von alleine einstellen, sondern die explizit und systematisch in einem guten Fachunterricht mit vermittelt werden müssen. Das betrifft sowohl das Mündliche wie das Schriftliche (Becker-Mrotzek et al. 2013, S. 7).

Auf das Fach bezogene Sprachkenntnisse kommen also dem fachlichen Lernen zugute zugleich können diese Kenntnisse nicht unabhängig vom Fach erworben werden. Sprachbildung muss daher im Fachunterricht selbst verortet sein. Die Diskussion um ,language across the curriculum“ ist nicht neu, und Sprachbildung gilt auch in Deutschland längst als anerkannte Aufgabe aller Fächer (MSJK 1999; Thürmann et al. 2010; Becker-Mrotzek et al. 2013). Die hohe bildungspolitische Bedeutung, die dem Thema zugemessen wird, spiegelt sich nicht zuletzt in der Verankerung in den Bildungsstandards der Fächer (KMK 2005, 2012). Wurde die Notwendigkeit von sprachlicher (Zusatz-)Bildung bzw. Sprachförderung ${ }^{2}$ zunächst vor allem mit Blick auf diejenigen Kinder und Jugendlichen gesehen, die Deutsch als Zweit- oder Fremdsprache sprechen, so hat sich mittlerweile die Erkenntnis durchgesetzt, dass Sprache für Lernprozesse von so großer Bedeutung ist, dass Sprachbildung in allen Fächern, über alle Bildungsetappen hinweg und für alle Schüler*innen erfolgen sollte (Gogolin und Lange 2010; Becker-Mrotzek et al. 2013; Benholz et al. 2015). Ziel ist es, durch den Auf- und Ausbau von fachbezogenen wie auch überfachlichen kommunikativen Fähigkeiten allen Lernenden eine aktive Teilhabe an schulischen Bildungsprozessen - und weit darüber hinaus an der Wissensgesellschaft (Ehlich 2015) - zu ermöglichen. Zu den zentralen Merkmalen eines sprachbildenden Fachunterrichts gehören ein verstehensorientierter Umgang mit Sprache und Mehrsprachigkeit im Fach sowie die systematische und individuelle sprachliche Unterstützung

2 Zur Unterscheidung von „Sprachbildung“ und „Sprachförderung“ verweisen wir auf Morris-Lange et al. (2016, S. 9): „Sprachbildung (bzw. sprachliche Bildung) ist als ein Oberbegriff zu verstehen, der alle Formen von gezielter Sprachentwicklung umfasst. Sprachbildung zielt darauf ab, die Sprachkompetenz aller Schülerinnen und Schüler zu verbessern, unabhängig davon, ob sie in Deutschland aufgewachsen oder neu zugewandert sind. Sprachbildung findet im Sprach- und Fachunterricht statt [...]. Sprachförderung bezeichnet eine spezielle Form von Sprachbildung. Zielgruppe sind Kinder und Jugendliche mit sprachlichen Schwierigkeiten, z. B. Geflüchtete, die Deutsch als Zweitsprache erlernen. Sprachförderung erfolgt sowohl im Regelunterricht als auch in gezielten Förderstunden (Schneider et al. 2012, S. 23).“ 
von Schüler*innen beim fachlichen Lernen (Gibbons 1998, 2015; Gogolin et al. 2011; Schmölzer-Eibinger et al. 2013; Beese et al. 2014).

Mit Blick auf die Lehramtsstudierenden als zukünftige Fachlehrerinnen und Fachlehrer bedeutet das: Sie müssen im Studium die Gelegenheit bekommen, sich nicht nur mit fachwissenschaftlichen und fachdidaktischen Aspekten ihrer Fächer, sondern auch mit dem Thema Sprachbildung auseinanderzusetzen. Ob, wie und in welchem Umfang die Vermittlung entsprechender sprachwissenschaftlicher und sprachdidaktischer Grundlagen in das Lehramtsstudium integriert ist, unterscheidet sich aufgrund der Bildungshoheit der Länder je nach Hochschulstandort. ${ }^{3}$ So reichen die Qualifizierungsmaßnahmen für Lehramtsstudierende im Bereich Sprachbildung von obligatorischen Modulen für Deutsch als Zweitsprache/Sprachbildung (z.B. in NRW) ${ }^{4}$ über freiwillige Zusatzqualifikationen und -zertifikate ${ }^{5}$ bis hin zur Möglichkeit, Deutsch als Zweitsprache als Unterrichts- oder Lehramtserweiterungsfach bzw. Studienschwerpunkt zu wählen. ${ }^{6}$

Übergeordnetes Ziel ist es, bei aller Unterschiedlichkeit der Angebote im Einzelnen, die Bewusstheit und die Kenntnisse der Studierenden im Bereich Sprache, Mehrsprachigkeit und Sprachbildung zu stärken und sie so auf den Umgang mit unterschiedlichen sprachlichen Voraussetzungen bei den Lernenden vorzubereiten. Zu den klassischen Inhalten von Veranstaltungen im Bereich Sprachbildung/Deutsch als Zweitsprache gehören daher:

- Erwerbsprozesse des Deutschen als Erst-, Zweit- und Fremdsprache

- Sprach(en)politik

- Konzepte von Mehrsprachigkeit

- Grammatische Grundlagen des Deutschen

- Sprachvergleich

- Alltags-, Bildungs- und Fachsprache

- Lehrmaterialanalyse

- Unterricht für neu zugewanderte Schülerinnen und Schüler

- Sprachstandsdiagnostik

- Lernersprachenanalyse, Fehleranalyse

- Ansätze und Methoden für Sprachbildung im Fach

Die Organisation und Durchführung der universitären Lehrveranstaltungen liegt i. d. R. im Verantwortungsbereich der Fächer Deutsch als Zweit-/Fremdsprache, Germanistik und/oder Bildungswissenschaften. Dadurch ist gewährleistet, dass die oben genannten

3 Die Bestandsaufnahmen zu Studienanteilen im Bereich Sprachförderung/Deutsch als Zweitsprache/Sprachbildung in der Lehramtsausbildung von Baumann und Becker-Mrotzek (2014), Morris-Lange et al. (2016) und Baumann (2017) dokumentieren die erheblichen Unterschiede in den formalen Vorgaben auf Länderund Hochschulebene.

4 Ob ein Nachweis in Deutsch als Zweitsprache im Lehramtsstudium verpflichtend ist, hängt in manchen Bundesländern vom gewählten Fach ab. Während etwa in Nordrhein-Westfalen Lehramtsstudierende aller Fächer einen Nachweis über „Kenntnisse in Deutsch als Zweitsprache“ erbringen müssen (MSW NRW 2009), trifft dies z. B. im Saarland verpflichtend nur auf Studierende im Lehramt Deutsch zu. Die Verortung im Studium (Bachelor und/oder Master) und die Anzahl der zu erbringenden Leistungspunkte variiert je nach Bundesland, teilweise finden sich auch Unterschiede zwischen Hochschulen im gleichen Bundesland.

5 Auch hier zeigt sich eine große Varianz in Umfang und entsprechend auch inhaltlicher Reichweite der Angebote.

6 Hierzu auch Witte (2017). 
Inhalte wissenschaftlich fundiert und in der gesamten Breite der Lehramtsausbildung vermittelt werden. Mit zunehmender Qualifizierung im Verlauf des Studiums und insbesondere im Kontext von Praxisphasen entsteht allerdings bei vielen Studierenden der Wunsch, den Zusammenhang zwischen Aspekten der Sprachbildung und „ihren“ Schulfächern genauer auszuloten. Die Notwendigkeit eines solchen dezidierten Fachbezugs verdeutlichen auch Projektzusammenhänge an der Schnittstelle von Forschung und universitärer Ausbildungspraxis. ${ }^{7}$ Im Rahmen der zentral gestalteten grundlegenden Studienangebote zum Thema Sprachbildung, die zunächst fachunabhängig angelegt sind, ist ein solch konkreter Bezug zu Inhalten und didaktischen Prinzipien der einzelnen Fächer (z. B. Mathematik, Geschichte, Informatik) jedoch schon aus organisatorischen Gründen nicht oder nur punktuell leistbar. Dafür sind Kooperationen mit den Fachdidaktiken notwendig, wie sie auch im Projekt „Deutsch als Zweitsprache in allen Fächern“ (ProDaZ; Universität Duisburg-Essen), in dessen Kontext auch das vorliegende Buch steht, umgesetzt werden. ${ }^{8}$ So lautet etwa eine Empfehlung des Projekts „Sprachen - Bilden Chancen“, dessen Ziel die Weiterentwicklung der Berliner Lehramtsausbildung im Bereich Sprachbildung/Deutsch als Zweitsprache war:

॥ [D]ie Bedeutung von Sprache für den Wissenserwerb im Allgemeinen und in einer jeweiligen fachdidaktischen Konkretisierung [sollte] noch stärker und - im Hinblick auf das Praxissemester - vor allem früher im Studienverlauf hervorgehoben werden. Vor diesem Hintergrund erscheint die strukturelle und inhaltliche Verankerung von Kooperationen mit den Fachdidaktiken sinnvoll (Sprachen - Bilden - Chancen 2018, 2 f.).

Auch aus fachdidaktischer Perspektive ist die Auseinandersetzung mit der Rolle von Sprache im eigenen Fach von großer Relevanz. So gilt etwa für das Fach Mathematik: Sprache ist in ihrer kommunikativen wie auch kognitiven Funktion ein wesentliches Element des mathematischen Lernens und insofern, wie oben dargelegt, ein wichtiger Forschungs- und Entwicklungsgegenstand der Mathematikdidaktik. Im universitären Alltag sind der Thematisierung von Aspekten der Sprachbildung im Mathematikunterricht allerdings häufig Grenzen gesetzt, auch weil eine Expertise zum Thema Sprachbildung zunächst vornehmlich in Fächern wie Deutsch als Zweit-/Fremdsprache verortet ist. Vor diesem Hintergrund ist auch aus fachdidaktischer Sicht eine entsprechende Kooperation sinnvoll und notwendig.

Der Fachbezug ist für die Studierenden von großer Bedeutung und eine Voraussetzung dafür, dass sie Sprachbildung in ihrem Unterricht auch tatsächlich umsetzen können.

7 Beispiele sind das hochschulübergreifende Berliner Projekt „Sprachen - Bilden - Chancen“, das „DaZKom“-Projekt der Universitäten Lüneburg und Bielefeld, das Projekt „ProfaLe“ an der Universität Hamburg, das Projekt „PSI“ an der Universität Potsdam oder die Projekte „ProViel“ und „ProDaZ“ an der Universität Duisburg-Essen.

8 Im Rahmen von ProDaZ sind seit 2009 interdisziplinäre Kooperationen unter anderem mit Kolleg*innen aus den Fachdidaktiken Geschichte, Physik, Chemie, Informatik, Mathematik u. v.m. entstanden. Die Ergebnisse der Zusammenarbeit sind in zahlreichen Publikationen dokumentiert (exemplarisch: Beese et al. 2014; Benholz et al. 2015; Beese et al. 2017; Roll et al. 2017; Moraitis et al. 2018; Roll et al. 2019a, b). Einen Überblick sowie über 300 Online-Publikationen zu den Themen Mehrsprachigkeit, Sprachentwicklung, Sprachstandsdiagnose, Sprachbildung und Sprachförderung bietet das Webportal des ProDaZ-Kompetenzzentrums ( $\triangleright$ https://www.uni-due.de/prodaz/kompetenzzentrum.php). 
Zwar bieten auch Veranstaltungen im Bereich Deutsch als Zweitsprache, Bildungswissenschaften oder Germanistik sowie Lehrpraktika Gelegenheiten, um Ideen zum sprachbildenden Fachunterricht zu entwickeln und zu erproben. Rückmeldungen wie die folgende zeigen aber, dass Studierenden die Übertragung von Inhalten aus dem Kontext Sprachbildung auf das eigene Fach nicht immer leichtfällt:

I) Das Thema Sprachbildung finde ich sehr interessant und wichtig. Ich frage mich aber, wie ich das in meinem Unterricht umsetzen kann. In einer DaZ-Veranstaltung haben wir z. B. das Scaffolding-Konzept kennengelernt, am Beispiel von Sachunterricht in der Grundschule. Aber wie kann ich das auf Mathematik in der Sekundarstufe übertragen? Und wie passt das mit den Prinzipien aus der Mathedidaktik zusammen? Manchmal habe ich das Gefühl, wir lernen überall etwas anderes - das überfordert mich (Lehramtsstudentin 2016).

Es besteht also die Gefahr, dass der Themenkomplex Sprachbildung trotz grundsätzlichen Interesses seitens der Studierenden nur schwer Eingang in die Unterrichtspraxis findet. Für die Lehramtsausbildung folgt daraus, dass die Studierenden sich zunächst der Bedeutung von Sprache in Lehr-Lern-Prozessen allgemein bewusst werden und Kenntnisse im Bereich sprachlicher Bildung erlangen. Darüber hinaus muss aber die spezifische Rolle von Sprache und Sprachbildung für die eigenen Unterrichtsfächer unter Bezug auf die jeweiligen Fachdidaktiken ausgelotet und praktisch erprobt werden:

I) Der Themenkomplex Sprachförderung und Deutsch als Zweitsprache sollte additiv und integrativ in die Hochschulausbildung einfließen: additiv als eigenes Sprachförder- und Deutsch-als-Zweitsprache-Modul und zusätzlich integrativ als Teil der Ausbildung in den Fachdidaktiken, Fach- und Bildungswissenschaften. (...) Die additiven Studienelemente sollten die sprachwissenschaftlichen und -didaktischen Grundlagen legen und auf die Rolle von Sprache in schulischer Bildung eingehen. In den Fächern sollte der Schwerpunkt auf dem Verhältnis von Sprache und Fach, geeigneten Diagnoseinstrumenten und Förderansätzen liegen (Baumann und Becker-Mrotzek 2014, S. 48 f.).

Zentrale Prinzipien aus den Bereichen (Zweit-)Sprachdidaktik/Sprachbildung und aus der jeweiligen Fachdidaktik sollten daher auf theoretischer Ebene wie auch in der Planung und Durchführung konkreter Unterrichtseinheiten aufeinander bezogen und ihre Synergien herausgearbeitet werden. Wenn die Studierenden analytisch und praktisch erfahren, wie sprachliches und fachliches Lernen in ihren Fächern zusammenhängen und sie geeignete Möglichkeiten zur Umsetzung von Sprachbildung nicht nur (theoretisch) kennenlernen, sondern auch selbst im forschenden Lernen erkunden, kann die Diskrepanz zwischen positiver Einstellung einerseits und unzureichender unterrichtlicher Umsetzung andererseits gelöst werden. Dafür sollte sich die für den Unterricht gewünschte Integration von fachlichem und sprachlichem Lernen idealerweise in der universitären Professionalisierung selbst spiegeln: Sprachbildung sollte in einer sprachbewussten Hochschullehre nicht nur ergänzend, sondern auch und vor allem integriert thematisiert werden. Insgesamt steht dabei das Ziel im Mittelpunkt, eine Bewusstheit für die vielfältigen Zusammenhänge von Sprache und Fach zu erzeugen und so auf einen sprachbildenden Unterricht vorzubereiten. 
Unter „Sprachbewusstheit“ (engl. language awareness) versteht man allgemein explizites Wissen über Sprache(n) sowie das bewusste Wahrnehmen und Umgehen mit Sprache und Mehrsprachigkeit im Lernprozess. Der Begriff wurde in Großbritannien in den 1970er Jahren zunächst im Kontext des Fremdsprachenlernens geprägt und fand u. a. Eingang in den Gemeinsamen Europäischen Referenzrahmen für Sprachen (Europarat 2001, 2020). Zugleich wurde der Begriff früh mit Blick auf die Berücksichtigung von Herkunftssprachen und Mehrsprachigkeit in den (Deutsch-)Unterricht diskutiert (im Überblick z. B. Luchtenberg 2002; Gürsoy 2010; Oomen-Welke 2016). Im Zuge von Forschungen zur Bedeutung von Sprache und Sprachbildung für das fachliche Lernen ist der sprachbewusste (auch: sprachsensible, sprachaufmerksame) Fachunterricht und eine entsprechende Qualifizierung von Lehrkräften in den Fokus gerückt (z. B. Leisen 2010, 2016; Schmölzer-Eibinger et al. 2013; Tajmel und Hägi-Mead 2017 uvm.). Das vorliegende Buch knüpft daran an und stellt die Frage in den Mittelpunkt, wie angehende Lehrkräfte auf sprachbewusstes Handeln im Fachunterricht Mathematik vorbereitet werden können. Entsprechend wird der Begriff sprachbewusste Hochschullehre verwendet, um die Integration von Fach und Sprache auf Ebene der universitären Lehramtsausbildung zu beschreiben. Eine sprachbewusste Hochschullehre schärft die Bewusstheit der Studierenden für die vielfältigen Beziehungen von sprachlichem und fachlichem Lernen - und zwar konkret mit Blick auf die Bedingungen des jeweiligen Fachs (vgl. A Abschn. 1.2.1). Fach und Sprache bilden damit eng aufeinander bezogene Lern- und Forschungsgegenstände, wobei Sprache bewusst als ein prägendes Merkmal fachlichen Denkens und Handelns adressiert wird (vgl. A Abschn. 1.2.2). Voraussetzung für eine sprachbewusste Hochschullehre ist die Herausarbeitung der Beziehungen von Fach und Sprache, z. B. im Kontext interdisziplinärer Kooperationen.

- Abb. 1.1 veranschaulicht die verschiedenen Schichtungen der Lehramtsausbildung, in denen die Vernetzung von Fach und Sprache zum Tragen kommt. Die gestrichelten Linien zeigen die Durchlässigkeit zwischen den unterschiedlichen Bereichen an; die Pfeile stehen für die Impulse, die von der Forschung, vom Unterricht und von der Lehre ausgehen und in die jeweils anderen Bereiche wirken können. So können beispielsweise studentische Beobachtungen während eines Schulpraktikums Impulse für Fragestellungen im Kontext des forschenden Lernens geben, die wiederum Gegenstand der gemeinsamen Diskussion im Seminar werden; der kontinuierliche interdisziplinäre Austausch und damit verbundene neue Erkenntnisinteressen verändern die thematischen Schwerpunktsetzungen in der gemeinsamen Forschung und Lehre usw.

Die Relevanz der Vernetzung von Fach und Sprache auf Hochschulebene wird im Folgenden an drei Beispielen für das Fach Mathematik erläutert ( Abschn. 1.1.1-1.1.3).

\subsubsection{Die Beziehung von fach- und sprachdidaktischen Konzeptbildungen für die Hochschullehre}

Das erste Beispiel bezieht sich auf die Frage, wie sich (zweit-)sprachdidaktische und fachdidaktische Konzeptbildungen für den Unterricht im Rahmen der universitären Ausbildung angehender Mathematiklehrkräfte sinnvoll aufeinander beziehen lassen. Dafür werden zwei ausgewählte, für die jeweilige Disziplin typische Lerngegenstände herangezogen: die Kernprozesse des Erkundens, des Systematisierens und des Übens für 


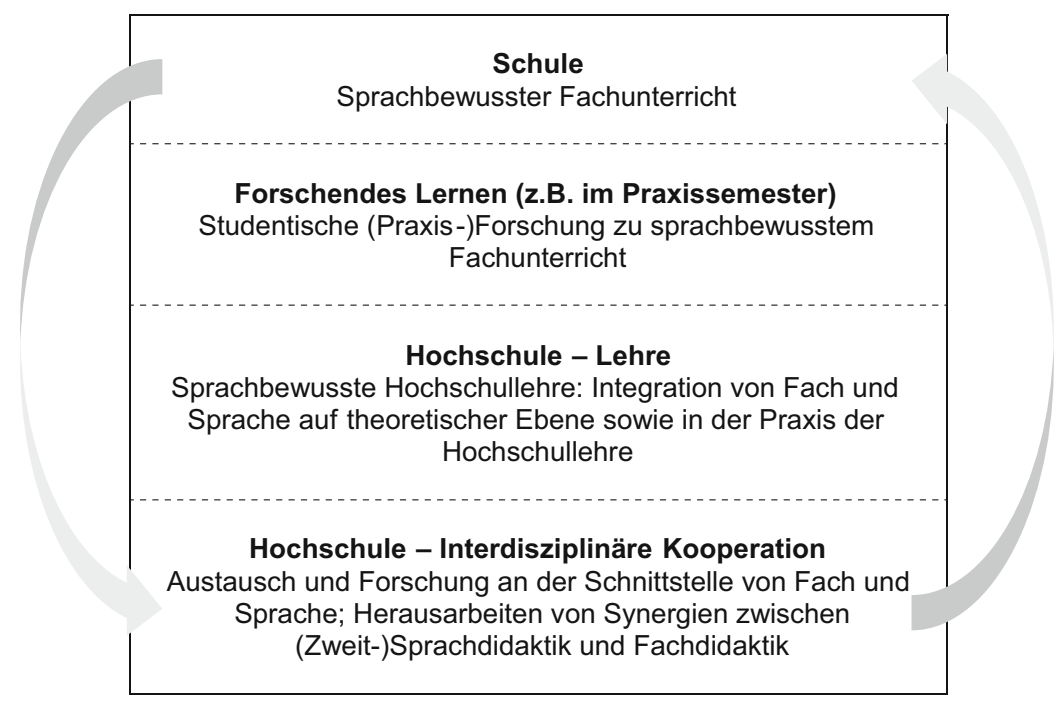

- Abb. 1.1 Vernetzung von Fach und Sprache in der Lehramtsausbildung

die mathematikdidaktische Perspektive, das Scaffolding-Modell für die zweitsprachdidaktische Perspektive. Im Anschluss an eine kurze Vorstellung der jeweiligen Konzeptbildungen wird exemplarisch diskutiert, wie diese in Beziehung gesetzt werden können. Dabei ist natürlich hervorzuheben, dass es sich um Lerngegenstände für die Hochschullehre handelt, d.h. die Studierenden sollten die Gelegenheit bekommen, Zusammenhänge selbst zu erarbeiten und verschiedene Varianten durchzuspielen. Auf der Ebene des Mathematikunterrichts können diese Konzeptbildungen dann leitend für professionelles unterrichtliches Handeln der Lehrperson sein.

\section{- Kernprozesse als universitärer Lerngegenstand (mathematikdidaktische Perspektive)}

In mathematikdidaktischen Lehrveranstaltungen werden i. d. R. Modelle thematisiert, die die Besonderheiten unterschiedlicher Phasen und damit verbundener Kernprozesse im Mathematikunterricht herausstellen. Dazu gehören etwa die Kernprozesse des Erkundens, des Systematisierens oder des vertiefenden Übens (z. B. Leuders et al. 2011; Hußmann 2003; vom Hofe 2001; Büchter und Leuders 2005, S. 118 f.; Prediger et al. 2011).

Der Kernprozess des Erkundens (bzw. des Entdeckens oder Erfindens) adressiert die Vorschauperspektive in Lernprozessen (z. B. Leuders et al. 2011; Hußmann 2003; vom Hofe 2001). Den Ausgangspunkt bilden dabei häufig mathematisch substanzhaltige Fragen oder Kernideen (zur Rolle der Kernideen etwa Ruf und Gallin 1998), die den weiteren Lernprozess strukturieren. Ausgelöst durch solche Kernideen entwickeln die Lernenden individuelle mathematische Ideen und begriffliche Zusammenhänge, die in der Regel zunächst auf präformalem Niveau bearbeitet werden. Solche Erkundungsprozesse sind üblicherweise durch eine recht hohe Offenheit z. B. hinsichtlich der Ausgangssituation, des Lösungsweges oder auch des Ergebnisses gekennzeichnet (Büchter und Leuders 2005, S. $118 \mathrm{f}$.).

Die Systematisierung der entwickelten individuellen Konzeptbildungen bedarf reflexiver Prozesse, die im weiteren Fortgang eine Anbindung zu den konsolidierten mathe- 
matischen Konzeptbildungen ermöglichen (z. B. Prediger et al. 2011). Zentral für solche Systematisierungsprozesse sind unterschiedliche Aneignungshandlungen der Schülerinnen und Schüler, die sich in der Art der Lernendenaktivierung und der gestellten Vorgaben deutlich unterscheiden können. So ist etwa die zusammenfassende Abschrift einer Definition im Mathematikbuch hochgradig konvergent, das heißt alle Schülerinnen und Schüler notieren die inhaltlich und sprachlich gleichgefasste Definition in ihren Heften. Gleichzeitig ist der Grad der individuellen kognitiven Aktivierung in diesem Beispiel denkbar gering. Im umgekehrten Fall formulieren Lernende im Nachgang einer Erkundungsphase (z. B. zu Funktionsgraphen ganzrationaler Funktionen) eigene Entdeckungen (z. B. im Rahmen von Hypothesen, Sätzen oder Definitionen). Der Grad der individuellen kognitiven Aktivierung ist hierbei vergleichsweise sehr hoch, während allerdings die sprachliche und inhaltliche Vergleichbarkeit i. d. R. stark schwankt. Im unterrichtlichen Alltag werden daher häufig Systematisierungsvarianten gewählt, bei denen die Aneignungshandlungen sich zwar an bestimmten Vorgaben orientieren, die aber gleichzeitig noch einen vergleichsweise hohen Aktivierungsgrad zulassen.

Der Kernprozess des vertiefenden Übens schließlich stellt eine (idealerweise produktive) Tätigkeit im Mathematikunterricht dar, bei der Möglichkeiten zum Trainieren, Problemlösen und Vernetzen von erarbeiteten Begriffen geboten werden (dazu z. B. Leuders 2009).

\section{- Scaffolding-Modell als universitärer Lerngegenstand (zweitsprachdidaktische Perspektive)}

Das Scaffolding-Modell wird in Lehrveranstaltungen im Bereich Deutsch als Zweitsprache/Sprachbildung häufig herangezogen, um zu veranschaulichen, wie Schüler*innen, die die Unterrichtssprache als Zweitsprache sprechen, im Fachunterricht systematisch unterstützt werden können.

Die Metapher des Scaffolding (wörtlich: Baugerüst) wurde zuerst von den Psychologen David Wood, Jerome Bruner und Gail Ross verwendet (Wood et al. 1976), um die elterliche Unterstützung von Kindern beim Problemlösen zu charakterisieren:

I) More often than not, it [the intervention of a tutor] involves a kind of „scaffolding“ process that enables a child or novice to solve a problem, carry out a task or achieve a goal which would be beyond his unassisted efforts. (...) The task thus proceeds to a successful conclusion. We assume, however, that the process can potentially achieve much more for the learner than an assisted completion of the task. It may result, eventually, in development of task competence by the learner at a pace that would far outstrip his unassisted efforts (Wood et al. 1976, S. 90).

Es handelt sich also um vorübergehende ${ }^{9}$ Unterstützungshandlungen, die Erwachsene in der Interaktion mit Kindern im Kontext gemeinsamer, interaktiv konstruierter Lernprozesse flexibel einsetzen. Die Unterstützungshandlungen ermöglichen es dem Kind nicht nur, Aufgaben erfolgreich zu bewältigen, die über seinem aktuellen Kompetenzniveau liegen, sondern können auch zu einer beschleunigten Aufgabenkompetenz führen. Das Scaffolding-Modell steht in engem Zusammenhang mit Lew Vygotskijs Theorie von der Zone der nächsten Entwicklung (ZNE) (Vygotskij 1934; Bruner 1985).

In der Zweitsprachdidaktik wurde die Scaffolding-Metapher aufgegriffen, um ein bedarfsgerechtes Unterstützungssystem für Schüler*innen zu beschreiben, deren Erst-

9 Daher die Metapher des Baugerüsts, das zurückgebaut wird, sobald das Bauwerk stabil ist. 
sprache sich von der dominanten Unterrichtssprache unterscheidet (z. B. Gibbons 1998, Hammond und Gibbons 2001, 2005; Gibbons 2015). Ziel ist es, allen Schülerinnen und Schülern eine aktive Teilhabe an anspruchsvollen fachlichen Lernprozessen zu ermöglichen:

I) $[\ldots]$ as far as possible, all learners, including second-language learners, need to be engaged with authentic and cognitively challenging learning tasks. It is the nature of the support customised support that is responsive to the needs of particular students - that is critical for success (Hammond und Gibbons 2001, S. 11).

Dies soll ausdrücklich nicht durch sprachliche oder inhaltliche Vereinfachungen geschehen; vielmehr werden die Schüler*innen durch eine geeignete Strukturierung von Unterricht, durch passgenaue (geplante oder spontane) Hilfen und nicht zuletzt durch eine unterstützende, ressourcenorientierte Grundhaltung seitens der Lehrkraft in die Lage versetzt, zunehmend sprachlich und kognitiv anspruchsvolle Aufgaben selbst erfolgreich zu bearbeiten:

I) (...) our concern is with ways of supporting-up such students, rather than with dumbingdown the curriculum (Hammond und Gibbons 2005, S. 6).

Den Rahmen bildet das sog. Makro-Scaffolding, das unter anderem die Auswahl und Sequenzierung von Aufgaben - unter Berücksichtigung der übergeordneten Lernziele sowie der Lernvoraussetzungen bei den Schüler*innen - betrifft. Dabei können zwei Prinzipien als Orientierung dienen (Gibbons 1998, 2015; Kniffka und Neuer 2008): Auf der fachlichen Ebene wird zunächst ein verstehens- und handlungsorientierter Zugang zum Fachinhalt ermöglicht - z. B. durch konkrete Anschauung in Experimenten; die Lernenden werden dann in mehreren Phasen zu einer inhaltlichen Verarbeitung auf höherem Abstraktionsniveau geführt. Auf der sprachlichen Ebene werden zunächst die alltagssprachlichen und mündlichen Fähigkeiten der Lernenden aktiviert; im Zuge der fachlichen Vertiefung werden dann systematisch ihre bildungs- und fachsprachlichen Fähigkeiten aufgebaut (sog. „,mode continuum“, Gibbons 2015). Dabei wird insbesondere dem Schreiben eine wichtige Rolle bei der Präzisierung und Strukturierung des fachlichen Denkens zugeschrieben (kognitive Funktion von Sprache). Entsprechend den Lernvoraussetzungen können geeignete Aktivitäten zur Unterstützung eingeplant werden, wie zum Beispiel die modellgeleitete Auseinandersetzung mit Fachtextsorten ${ }^{10}$, ausgewählte Formen der Kooperation unter den Schüler*innen, die Vernetzung unterschiedlicher Darstellungen ${ }^{11}$ oder die angeleitete Reflexion von Lernprozessen. ${ }^{12}$

Einen besonderen Stellenwert nimmt die spontane, interaktionale Unterstützung im Unterrichtsdiskurs ein, bei der die Lehrkraft lokal auf die jeweiligen sprachlichen Bedürfnisse der Schüler*innen reagiert (sog. Mikro-Scaffolding):

I) The key element of micro scaffolding (...) is the contingent nature of support. The teacher is constantly monitoring students' understanding and ability in order to determine the minimum support required. In response, the teacher is constantly removing or supplying support as needed to complete the task at hand (Dansie 2001, S. 50).

10 „,teaching and learning cycle“, Gibbons (2015); Gürsoy (2018); Roll et al. (2019a).

11 Wessel (2015); Abschn. 2.3.

12 Einen Überblick bietet Kniffka (2010). 
Voraussetzung für eine solche nicht (im Detail) planbare Unterstützung ist, dass die Lehrkraft über eine hohe (fach-)sprachliche Bewusstheit verfügt und die Kommunikation mit und unter den Schüler*innen aufmerksam begleitet. ${ }^{13}$

Im Rahmen einer sprachbewussten Hochschullehre ist nun zu diskutieren, in welchem Verhältnis - beispielsweise - die Prinzipien des Scaffolding und das in der Mathematikdidaktik vermittelte Modell der Kernprozesse stehen und welchen Gewinn die Verknüpfung von Fach- und Sprachdidaktik für die sprachbewusste Planung und Durchführung von Unterricht mit sich bringt. So ist etwa eine in Lehrveranstaltungen häufig gestellte Frage, wie sich die sprachliche Unterstützung im Rahmen offener Erkundungen im Unterschied zu Phasen des Systematisierens oder Phasen des Übens gestalten lässt. Hierin liegt u. E. ein entscheidender Punkt: Wenn die Studierenden sich in universitären Lehrveranstaltungen (und darüber hinaus in Praxisphasen) aktiv mit der Frage auseinandersetzen, wie sich zentrale fachdidaktische Konzepte ihrer jeweiligen Fächer und (zweit-)sprachdidaktische Konzepte zueinander verhalten und wo sich Synergien ergeben, so kann im späteren Schulalltag die Umsetzung eines sprachbewussten Fachunterrichts leichter gelingen.

Zur Veranschaulichung und kritischen Diskussion in der Lehre ist es sinnvoll, ein konkretes Unterrichtsbeispiel wie das folgende aus dem Mathematikunterricht einer 10. Klasse heranzuziehen. - Abb. 1.3 (am Ende des Abschnitts) fasst das Beispiel zusammen und zeigt die fach- und sprachdidaktischen Bezugspunkte auf.

\section{- Beispiel „Funktionsgleichungen"14}

In der Mathematikstunde einer 10. Klasse werden ganzrationale Funktionen im Rahmen einer offenen Erkundungssituation thematisiert. Die Schülerinnen und Schüler erhalten zunächst von der Lehrkraft mehrere ausgewählte Funktionsgleichungen ganzrationaler Funktionen (z. B. $f(x)=x^{2}+5 x$ ), die sie in Partnerarbeit mit dem Funktionenplotter graphisch darstellen (- Abb. 1.2) ${ }^{15}$.

Während der Partnerarbeit tauschen sich die Schüler*innen S1, S2, S3 und S4 über die Veränderungen aus, die sich ergeben, wenn sie Parameter der Funktionsgleichung eigenständig variieren:

(s01) S1: Das erste wird zusammengestreckt ((Geste mit Daumen und Zeigefinger „schmal nach oben“))

(s02) und das ist so gerade.

(s03) S2: Das wird gedrückt sozusagen ((Gestik))

(s04) ( ) [ Äußerung auf Türkisch]

(s05) S3: Wenn das bei 1 wäre, wäre es gestreckt.

(s06) S4: Das verschiebt sich nach rechts.

Die Schüler*innen befinden sich bei der Partnerarbeit im gemeinsamen Wahrnehmungsraum und können daher bei ihren Beschreibungen auf deiktische Mittel - hier insbesondere das Zeigwort „das“ (in (s02), (s03), (s05) und (s06)) sowie Gestik (in (s01) und (s03)) - zurückgreifen. Das Sich-Verlassen auf deiktische Mittel ist, anders als in der schriftlichen Kommunikation, in der mündlichen face-to-face-Situation unproblematisch und erleichtert im direkten Austausch die erste Annäherung an die fachlichen Sachverhalte.

13 Beispiele für eine unterstützende Gesprächsführung im Fachunterricht finden sich z. B. bei Leisen (2019).

14 Die Daten wurden im Kontext einer Schulkooperation an einem Gymnasium im Ruhrgebiet erhoben.

15 Die Funktionsgraphen wurden mit der Software GeoGebra erstellt. 


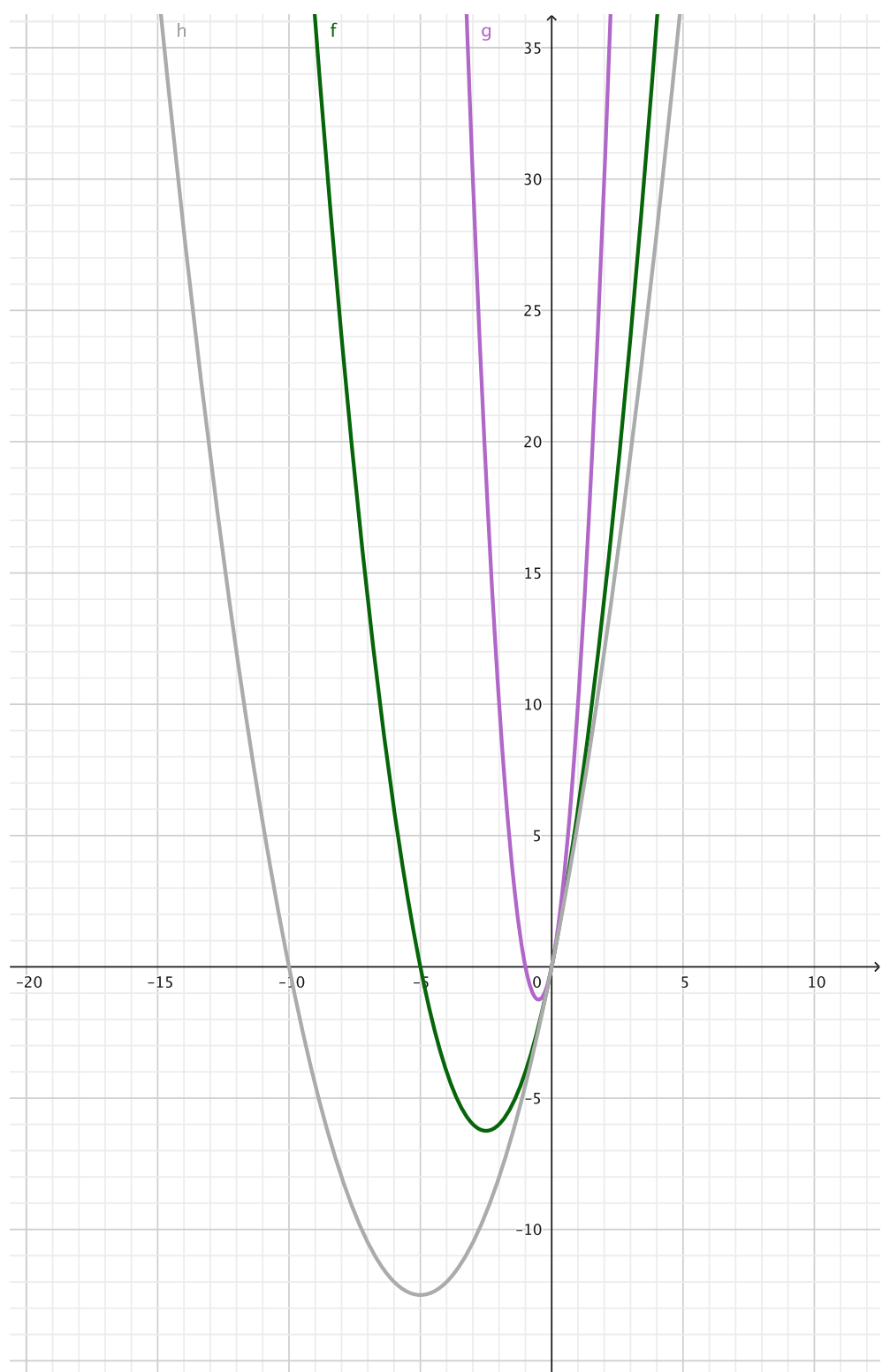

- Abb. 1.2 Funktionsgraphen $f, g$ und $h$ der ganzrationalen Funktionen mit $f(x)=x^{2}+5 x, g(x)=5 x^{2}+5 x$ und $h(x)=0,5 x^{2}+5 x$

Um die Veränderungen an den Funktionsgraphen zu beschreiben, nutzen die Schüler*innen teilweise bereits fachsprachliche Verben (,strecken“ (s05), „sich verschieben“ (s06)). Allerdings gelingt dies noch nicht immer: So versucht beispielsweise S2, mithilfe des Verbs „drücken“ (s03) eine Stauchung zu beschreiben. ${ }^{16}$ Zur Verständnissicherung

16 Indem er das Adverb „,sozusagen“ hinzufügt (,,wird gedrückt sozusagen“), macht er aber deutlich, dass er sich der Nicht-Angemessenheit des Ausdrucks ,,drücken“ im gegebenen Fachkontext durchaus bewusst ist. 
für den Lernpartner unterstützt er seine Beschreibung zum einen durch Gestik, zum anderen durch eine (nicht genauer dokumentierte) Ausführung auf Türkisch.

S3 geht über die reine Beschreibung von Beobachtungen hinaus und stellt eine Hypothese auf (s05).

Nachdem die Schüler*innen die entsprechenden Funktionsgraphen mithilfe eines Funktionenplotters graphisch dargestellt haben, sollen sie die Funktionen sowie die entsprechenden Funktionsgraphen auf Regelmäßigkeiten hin untersuchen und diese in Form von Hypothesen mit ersten Begründungsansätzen notieren. In der anschließenden Diskussion im Plenum geben sie ihre Beobachtungen wieder; die Lehrkraft unterstützt bei der Präzisierung und Systematisierung:

(s07) S5: Mir ist aufgefallen, dass wenn zum Beispiel eine 5 davor steht, dann wird der Graph schmaler ((Geste, bei der sich Daumen und Zeigefinger horizontal aufeinander zubewegen))

(s08) L: Also du meinst zum Beispiel $5 x^{2}+5 x$ ?

(s10) S5: Ja, genau.

(s11) L: Richtig, wenn ich zur Funktion $f(x)=x^{2}+5 x$ einen Vorfaktor bei $x^{2}$ hinzufüge, der größer als 1 ist, dann wird der Graph gestreckt.

(s12) Was passiert denn, wenn der Vorfaktor bei $x^{2}$ kleiner als 1 ist?

(s13) Also zum Beispiel 0,5 $x^{2}+5 x$ ?

(s14) S6: Dann wird es breiter.

(s15) L: Ja ((zögernd)) man könnte fast meinen, der Graph wird breiter.

(s16) Wenn wir einen Vorfaktor kleiner 1 haben, wird der Graph gestaucht.

Der Lehrer greift also zum einen die Schüleräußerungen auf und reformuliert sie mit Hilfe zentraler Fachbegriffe (,einen Vorfaktor hinzufügen“ (s11), „strecken“ (s11), „stauchen“ (s16)). Insofern ist er fachsprachliches Vorbild für die Lernenden. Zum anderen nimmt er eine inhaltliche Systematisierung vor, indem er die symbolische, die graphische und die verbale Darstellung miteinander vernetzt. Im weiteren Verlauf entwickelt sich eine Diskussion, inwiefern die Streckungen bzw. Stauchungen eher in vertikaler als in horizontaler Weise zu deuten sind und wie sich das mathematisch $-z$. B. über eine punktweise Betrachtung der Auswirkung der Stauchung bzw. Streckung auf den Funktionsgraphen begründen lässt. Es werden also die fachlich zum Teil nicht tragfähigen individuellen Vorstellungen der Schüler*innen (,,dann wird der Graph wird schmaler“ (s07); „Dann wird es breiter" (s14)) aufgegriffen und mit der konsolidierten Mathematik abgeglichen. Im Anschluss daran formulieren die Lernenden in Kleingruppen jeweils einen entsprechenden mathematischen Satz mit einem Beweis, der dann noch einmal zwischen den Gruppen ausgetauscht und hinsichtlich inhaltlicher und fachsprachlicher Aspekte beurteilt wird.

Dieses sehr knappe Unterrichtsbeispiel zeigt exemplarisch zwei Kernprozesse, in die die Schüler*innen involviert waren: zunächst den Prozess des Erkundens von Zusammenhängen ganzrationaler Funktionen mit entsprechender Dokumentation und anschließend den Prozess des Systematisierens im Rahmen einer Plenumsphase. Die kognitive Aktivierung der Lernenden ist in der Erkundungsphase hoch, insofern sie vor der Aufgabe stehen, die Funktionen sowie die dazugehörigen Funktionsgraphen auf Regelmäßigkeiten hin zu untersuchen. Dazu werden Prototypen herausgearbeitet, systematische Variationen vorgenommen und eigene Schwerpunkte gesetzt (z. B. hinsichtlich der Rolle von Streckfaktoren). Dem Kommunizieren und dem Argumentieren kommt dabei eine besonders wichtige Funktion zu, weil die zunächst eher experimentell ermittelten Ergebnisse 


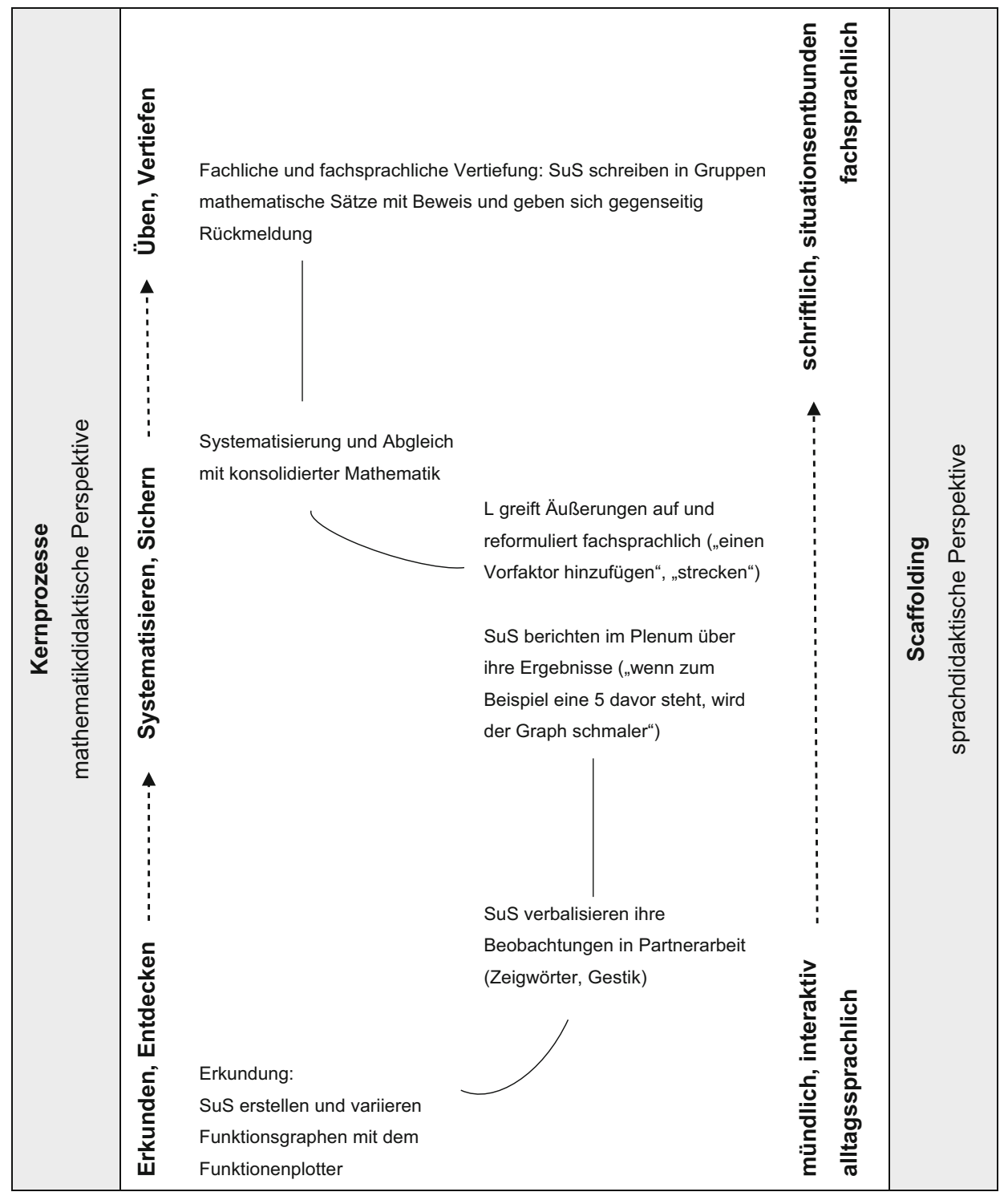

- Abb. 1.3 Verknüpfung fach- und sprachdidaktischer Konzepte

sprachlich gefasst und in entsprechende fachsprachliche Zusammenhänge (z. B. im Kontext des Streckens und Stauchens) eingeordnet werden müssen. Dies geschieht - im Sinne des Scaffoldings - in dem obigen Beispiel zunächst auf mündlicher, dann auf schriftlicher Ebene, wobei für diese Phasen jeweils auch soziale Aushandlungsprozesse sowohl inhaltlicher als auch sprachlicher Art charakteristisch sind.

Die mathematikdidaktischen bzw. sprachdidaktischen Konzepte erlauben dabei eine sich ergänzende Sicht auf die zugrundeliegenden fachlichen und sprachlichen Prozesse, wie in - Abb. $1.3^{17}$ zusammenfassend dargestellt. 
Das Beispiel „Funktionsgleichungen“ zeigt, wie theoretische Ansätze der jeweiligen Bezugsdisziplinen zur fach- und sprachintegrierten Analyse und Gestaltung unterrichtlicher Situationen beitragen können, wenn sie sinnvoll aufeinander bezogen werden. Damit soll natürlich nicht nahegelegt werden, dass Unterricht immer einem bestimmten Ablauf folgt oder folgen sollte; vielmehr kann in der Hochschullehre an diesem und vergleichbaren Beispielen zum einen diskutiert werden, wie Sprachbildung bei der fachbezogenen Unterrichtsplanung berücksichtigt werden kann und welche Variationsmöglichkeiten sich dabei bieten, z. B.:

- Wie können Aufgaben sinnvoll ausgewählt und sequenziert werden?

- Welche fachlichen und sprachlichen Anforderungen und Erwartungen sind mit den jeweiligen Arbeitsaufträgen verbunden?

- Welche Unterstützung ist in welchen Phasen sinnvoll?

- Wie können Aufgaben(teile) so variiert werden, dass sie mehr Unterstützungspotential bieten?

- Welche Rolle kann Darstellungsvernetzung spielen ${ }^{18}$

- Inwiefern kann an Alltagswissen angeknüpft werden?

Zum anderen lässt sich herausarbeiten, an welchen Stellen eine Vorab-Planung nicht möglich ist, wo also ein flexibles Eingehen auf schülerseitige Beiträge im Unterrichtsdiskurs im Sinne spontanen, interaktiven Mikro-Scaffoldings notwendig ist ( Abschn. 1.2.1, Leitidee L3). Beides kann gelingen, wenn die Studierenden eine hohe Bewusstheit für Sprache im Fach ausbilden, also lernen, Sprache in fachlichen Zusammenhängen kontinuierlich mitzudenken ( Abschn. 1.1.3).

\subsubsection{Der Zusammenhang von Denken und Sprechen im Studium}

Während in Abschn. 1.1.1 die Beziehung mathematik- und sprachdidaktischer Konzeptbildungen für den Unterricht als Lerngegenstände der Hochschullehre im Mittelpunkt steht, soll im Folgenden die Rolle von Denken und Sprechen sowie deren Relevanz für Studierende genauer diskutiert werden.

Die Beziehung von Denken und Sprechen fasziniert den Menschen seit jeher. Ein Grund ist sicher darin zu sehen, dass die Sprache und das Denken in Begriffen spezifisch für uns Menschen sind. Für die Beschäftigung mit Mathematik ist diese Beziehung insofern besonders spannend, als Kognition und Sprache nicht voneinander getrennt werden können, weil die Herausarbeitung (neuer) begrifflicher Beziehungen Kernelement mathematischen Handelns ist. So ist auch Wittenberg (1968) zu verstehen, der Mathematik als ein „Denken in Begriffen“ bezeichnet.

Nach Kant ist „Denken (... ) das Erkenntniß durch Begriffe“ (Kant 1999, S. 134). Mit der Kritik der reinen Vernunft entwickelt Kant u. a. ein bis dahin völlig neues Verständnis der Beziehung von Denken und Sprechen. Die begriffliche Struktur der Sprache ist demnach nicht als Spiegel der Welt zu verstehen. Es ist vielmehr so, dass der Mensch die Welt mit Begriffen überhaupt erst strukturiert und begreift. Auch Vygotskij (1934/2002) diskutiert in seinem Buch Denken und Sprechen die Frage, inwiefern diese beiden Prozesse miteinander zusammenhängen. Für Vygotskij sind sie in einer dialektischen Weise eng miteinander verknüpft. Das heißt auch: Weder das Sprechen noch das Denken übernimmt

18 Zu Darstellungsvernetzung und Scaffolding s. Wessel (2015). 
dabei eine prioritäre Rolle. Vygotskij wendet sich gegen die Annahme, dass Sprache letztlich nur die (bereits fertigen) Gedanken zum Ausdruck bringt. Vielmehr vollzieht sich nach Vygotskij - in dialektischer Weise - das Denken im Sprechen: „Das Sprechen ist seiner Struktur nach keine spiegelhafte Abbildung der Struktur des Denkens. (... ) Indem sich der Gedanke in Sprechen verwandelt, gestaltet er sich um, verändert er sich. Der Gedanke drückt sich im Wort nicht aus, sondern vollzieht sich im Wort." (Vygotskij 1934/2002, S. 401)

Diese hoch interessante Frage nach dem Verhältnis von Denken und Sprechen wird nicht nur in philosophischen Fachkreisen sehr intensiv diskutiert, sie spielt auch im Alltag der Hochschullehre eine wichtige Rolle. Dies zeigen exemplarisch die Kommentare zweier Studierender, die sich im Rahmen eines Seminars anhand von schriftlichen Schülerbeispielen mit einer Lernausgangsdiagnose zu negativen Zahlen beschäftigen:

1) „Bei den Schülerantworten zu der Frage, welche der Zahlen -5 und -3 größer ist, kann ich die unterschiedlichen Schülervorstellungen kategorisieren - etwa dahingehend, wie sich die Schüler die Anordnung der Zahlen vorstellen. Aber das hat doch nichts mit Sprache zu tun!“

2) „Die sprachliche Gestaltung der Schülerantworten zur Frage nach der Anordnung von Zahlen kann ich analysieren. Aber ich frage mich, inwiefern mir das für die Untersuchung des mathematischen Verständnisses helfen soll!“‘

Die Studierenden erkennen also jeweils den Wert der sprachlichen bzw. fachlichen Analyse der Lernendenprodukte. Gleichzeitig hinterfragen sie, inwiefern diese beiden (sprachlichen und fachlichen) Perspektiven miteinander zusammenhängen.

Die Herausforderung im Alltag der Hochschullehre besteht für die Lehrenden häufig darin, die Bedeutung einer gemeinsamen Perspektive auf schulische Lernendenprodukte erfahrbar zu machen. Eine solche gemeinsame Perspektive zeichnet sich mit der vorliegenden Konzeption einer sprachbewussten Hochschullehre durch die Verknüpfung von sprachlichen und mathematikdidaktischen Analyseansätzen aus, wenngleich sich die Konzepte auch auf weitere Domänen übertragen lassen (vgl. \ Kap. 3).

Inwiefern der in den beiden Studierendenäußerungen zum Ausdruck gebrachte Widerspruch aufgelöst werden kann, soll das folgende Beispiel verdeutlichen.

\section{- Beispiel: Negative Zahlen}

Für eine Lernausgangsdiagnose in einer Klasse 6 zum Thema negative Zahlen hat eine Studierende unterschiedliche Aufgaben zusammengestellt, von denen einige die Anordnung der ganzen Zahlen adressieren. Ziel der Erhebung war es im weiteren Sinne, die Vorstellungen von Schülerinnen und Schülern zu negativen Zahlen genauer zu untersuchen. Für viele Schüler*innen stellen negative Zahlen eine begriffliche Herausforderung dar:

॥ Negative numbers are often viewed as unsolvable mysteries by many students (Mukhopadhyay 1997, S. 35).

Von entscheidender Bedeutung in diesem Zusammenhang ist ein inhaltliches Verständnis der Ordnungsrelation, d. h. darüber,

॥ wann etwas kleiner oder größer ist. Die Ordnungsrelation ist diesbezüglich bei der Zahlbereichserweiterung grundlegend. (...) Die Entwicklung einer Ordnungsrelation für ganze Zahlen, welche sowohl die bekannten natürlichen, als auch die ,neuen“ negativen ganzen Zahlen umfasst, stellt (...) jedoch eine Herausforderung dar (Schindler 2014, S. 78). 
Im Rahmen der Erhebung wurde bewusst entschieden, dass die Lernenden die begrifflichen Zusammenhänge auch sprachlich reflektieren und nicht allein Fertigkeiten in Bezug auf den Kalkül bzw. die zugrunde liegenden Operationen untersucht werden. Für Zahlbereichserweiterungen (z. B. von den natürlichen Zahlen $\mathbb{N}$ zu den ganzen Zahlen $\mathbb{Z}$ ) besonders wichtig ist ein sicheres Verständnis der Ordnungsrelation: Die ganzen Zahlen $\mathbb{Z}$ lassen sich der Größe nach anordnen, was sich z. B. mit Hilfe der Zahlengeraden von links nach rechts bzw. von unten nach oben auch visuell darstellen lässt. Diese Eigenschaft ist übrigens nicht selbstverständlich für Zahlen - so lassen sich etwa die komplexen Zahlen $\mathbb{C}$ nicht entlang einer Zahlengeraden anordnen.

Die Studierende ließ die Schülerinnen und Schüler im Rahmen ihres Studienprojekts folgende Aufgabe bearbeiten:

$-5>-3$

Entscheide, ob die Ungleichung richtig oder falsch ist. Begründe deine Entscheidung.

Die Studierende hat bei der Gestaltung der Lernausgangsdiagnose zwei Aspekte besonders berücksichtigt: Zum einen sollen die Lernenden zur Sprachproduktion angeregt werden, indem sie etwa Begründungen erarbeiten, Hypothesen aufstellen oder Beurteilungen vornehmen. Dies erfolgte in der Aufgabe oben etwa so, dass die Lernenden entscheiden sollen, ob die Ungleichung $-5>-3$ richtig oder falsch sei. Außerdem sollten sie eine entsprechende Begründung für ihre Einschätzung abgeben. Der zweite Aspekt hinsichtlich der Gestaltung der Lernausgangsdiagnose war die Verwendung unterschiedlicher Repräsentationsmittel im Rahmen der Erhebung. Der fachliche Hintergrund ist darin zu sehen, dass das Verständnis mathematischer Begriffe ganz wesentlich damit zusammenhängt, unterschiedliche Darstellungen von Begriffen miteinander zu vernetzen. Im vorliegenden Fall etwa sind neben der symbolischen Darstellungsform (Ungleichung: $-5>-3$ ) die ikonische (Zahlengerade) und die verbale (z. B. bei Begründungen) Darstellungsform besonders relevant.

Bei der Analyse der Schülerantworten fielen der Studierenden zwei Antworten auf:

a) „Die Aufgabe $-5>-3$ ist richtig, weil 5 größer als 3 ist.“ Dieses Beispiel dokumentiert eine typische Schwierigkeit im Umgang mit negativen Zahlen, bei der die Vorzeichen ignoriert werden. Empirisch sind durchaus recht unterschiedliche Vorstellungen von Lernenden dokumentiert, die als Begründung für eine solche Einschätzung herangezogen werden (etwa vom Hofe und Hattermann 2014; Schindler 2014; Rütten 2016). Diese reichen von Begründungsmustern im Sinne einer umgekehrten Ordnungsrelation bei negativen Zahlen bis hin zur Argumentation mithilfe des Modells einer geteilten Zahlengeraden.

b) „Die Aufgabe ist falsch, weil -3 näher an den Plus Zahlen steht.“ Dieses Beispiel dokumentiert, inwiefern Lernende im Rahmen ihrer Begründungen auf entsprechende Repräsentationen (hier etwa die Zahlengerade) zurückgreifen. Die Schülerin teilt die Zahlengerade in einen positiven und einen negativen Bereich. Vom Modell einer geteilten Zahlengeraden (divided number line model, Peled et al. 1989; Mukhopadhyay 1997) spricht man, wenn Lernende den Abstand der Zahlen von der Null als Anhaltspunkt dafür nehmen, die Größe von Zahlen miteinander zu vergleichen. Vergleicht man zwei negative Zahlen a und b, so ist die Zahl mit dem geringeren Abstand zur Null die größere Zahl. Vergleicht man hingegen zwei positive Zahlen $\mathrm{c}$ und d, so ist die Zahl mit dem größeren Abstand zur Null die größere Zahl. 
Ein stark regelgeleitetes Vorgehen im Sinne von b) ist allerdings nicht unproblematisch, etwa dann, wenn man eine positive mit einer negativen Zahl vergleicht, etwa -5 und 3. Eine Argumentation über den Abstand zur Null führt für den Vergleich der Größe der beiden Zahlen nicht weiter. Winter (1989b) formuliert diesen Zusammenhang so:

I) a kommt vor b, bzw. a ist kleiner als b, genau dann, wenn beim Zählen von 0 aus zuerst „,a“ und dann „, unten/links gezählt [...], so wird gerade die umgekehrte Sicht zugemutet: a kommt vor b bzw. a ist kleiner als b genau dann, wenn beim Zählen von 0 aus zuerst „, "“ und dann „a“ aufgezählt (genannt) werden. Solange das Zählen in dieser Weise auf den Nullpunkt zentriert ist, solange muß die Ordnung der negativen Zahlen widernatürliche Züge tragen (Winter 1989b, S. 23).

Für das Verständnis der ganzen Zahlen (d.h. der negativen ganzen und der natürlichen Zahlen) kommt es entscheidend darauf an, das Modell der geteilten Zahlengerade durch ein Modell der einheitlichen Zahlengeraden zu ersetzen (dazu etwa Schindler 2014; Rütten 2016; sowie zum continous number line model in Peled et al. 1989; Mukhopadhyay 1997). Eine künstliche Teilung der Zahlengeraden an der Null kann in diesem Zusammenhang zu erheblichen inhaltlichen Schwierigkeiten führen, denen sich aber mit entsprechenden fachdidaktisch fundierten Ansätzen begegnen lässt: „Erst die Vorstellung, das Vorwärts-Zählen durchgehend von unten/links nach oben/rechts zu betreiben, bringt es wieder mit der Ordnung in Einklang“ (Winter 1989b, S. 23).

Im Folgenden soll nun der Zusammenhang von Denken und Sprechen im Mittelpunkt stehen, der sich darin zeigt, wie die Studierende ihren analytischen Fokus verändert, mit dem sie die Schülerprodukte (a) und (b) betrachtet. Ursprünglich wollte sie die Sprachmittel genauer untersuchen, die die Lernenden bei dieser Aufgabe verwenden (sprachliche Perspektive). Im weiteren Verlauf dann wollte sie untersuchen, inwiefern die Lernenden bei ihren Begründungen auf unterschiedliche Darstellungen zurückgreifen und diese verwenden (fachliche Perspektive). Vor dem Hintergrund der beiden Schülerantworten (a) und (b) hat die Studierende ihren analytischen Fokus im Prozess verschoben. Im Mittelpunkt steht schließlich die Frage, welche Sprachmittel die Lernenden im Zusammenhang mit der Verwendung spezifischer Darstellungsformen nutzen. Dieser Fokus verknüpft die sprachliche und die fachliche Analyse in einer Weise, die die gegenseitige Abhängigkeit von sprachlicher und fachlicher Gestaltung der Lernendenprodukte genauer in den Blick nimmt. So zeigt etwa Antwort (a), dass im Zusammenhang mit der symbolischen Darstellung (Ungleichung: $-5>-3$ ) das Konzept der Größe aufgerufen wird, das sich als spezifisch (und als notwendig) für den Zahlvergleich erweist - hier in Form des Adjektivs ,groß“ im Komparativ: „weil 5 größer als 3 ist“. Anders bei der Verwendung der Zahlengeraden, einer ikonischen Darstellungsform, in Antwort (b): Hier wird mit dem Adjektiv „nah“ im Komparativ das Konzept der Entfernung aufgerufen, um die Position der Zahlen auf der Zahlengerade vergleichend zu beschreiben (,weil -3 näher an den Plus Zahlen steht"). Die Verwendung der Sprachmittel ist insofern auf das Engste mit der spezifischen Verwendung der Darstellungsformen und daher mit inhaltlichen Aspekten verknüpft. Diese Analyse hat der Studierenden Einsichten verschafft, die gerade die Beziehungen und die Abhängigkeiten von sprachlichen und fachlichen Aspekten betreffen. Möchte man solche Einsichten dann wiederum für die Gestaltung von Unterricht nutzen, so spielt die Verstehensorientierung eine entscheidende Rolle. Nach Prediger (2013) 
zeichnet sich ein verstehensorientierter Mathematikunterricht insbesondere dadurch aus, dass etwa Rechenwege mündlich und schriftlich verbalisiert werden, dass ein konzeptuelles Verständnis mathematischer Konzepte aufgebaut und gefördert wird und dass die jeweiligen Bedeutungen erklärt werden.

Im Hochschulalltag ist es eine besondere Herausforderung, fachliche Inhalte, die auch noch mit unterschiedlichen fachlichen Domänen - hier: Sprache und Mathematik - verknüpft sind, für die Betrachtung von konkreten Gegenständen (z. B. Lernendenprodukten) miteinander in Beziehung zu setzen. Die folgenden beiden (Forschungs-)Fragen, die Studierende vor dem Hintergrund der Analyse von Lernendenprodukten entwickelt haben, sind zwei passende Beispiele dafür, inwiefern eine solche gemeinsame Betrachtung fachlicher und sprachlicher Aspekte zu Einsichten führen kann, die bei getrennten Analysen in der Form möglicherweise nicht hätten herausgearbeitet werden können:

- Welche Sprachmittel nutzen Lernende in Abhängigkeit von der Verwendung unterschiedlicher mathematischer Darstellungsformen?

- Welche Sprachmittel nutzen Lernende im Zusammenhang mit spezifischen (Grund-) Vorstellungen, die in den Lernendenprodukten rekonstruierbar sind?

Diese beiden Fragen zeigen ganz praktisch, wie Studierende Denken und Sprechen miteinander in Beziehung setzen (können), wenn sie Lernendenprodukte genauer analysieren. Die Beispiele zeigen aber auch, dass die philosophisch sehr spannende Frage nach dem Verhältnis von Denken und Sprechen im Universitäts- und im Schulalltag gleichermaßen eine Herausforderung darstellt, die von Studierenden und Lehrkräften gelöst werden muss.

\subsubsection{Zur Bedeutung von Sprache für das Fach Mathematik}

Sprachbildender Unterricht soll die vorhandenen sprachlichen Fähigkeiten der Schüler*innen aufgreifen, ihre Bewusstheit für Sprache stärken und darüber hinaus zur aktiven Nutzung und Vernetzung der verfügbaren Ressourcen in den Erst-, Zweit- und Fremdsprachen im Unterricht anregen. Was auf den ersten Blick primär als Aufgabe der sprachlichen Fächer - also des Deutsch-, DaZ-, Fremdsprachen- und Herkunftssprachenunterrichts - erscheinen mag, erweist sich bei genauerer Betrachtung als ein Anliegen, das auch für ein Fach wie Mathematik von großer Bedeutung ist. Denn gerade in mathematischen Zusammenhängen ist der präzise Umgang mit Sprache häufig ausschlaggebend für das konzeptuelle Verstehen und damit für erfolgreiches fachliches Lernen. Entsprechend beschäftigt sich auch die Mathematikdidaktik ,seit ihrer Entstehung mit sprachlichen und kommunikativen Aspekten des Mathematiklernens als Lerngegenstand, Lernmedium und Lernvoraussetzung “ (Prediger 2013, S. 161). In diesem Zusammenhang werden - gerade im Rahmen der Ausbildung - die unterschiedlichen Funktionen von Sprache thematisiert (vgl. Meyer und Tiedemann 2017, S. 39 ff.). Die kognitive Funktion der Sprache spielt beim Erkenntnisgewinn eine Rolle, z. B. indem Sprache begriffliches Wissen strukturiert. Ein Beispiel: Jedes Quadrat ist ein Rechteck und jedes Rechteck ein Viereck. Zugleich hat Sprache eine kommunikative Funktion, die dem Austausch, dem Diskurs und der Explizierung dient. Indes lassen sich diese Funktionen kaum voneinander trennen, weil sie ,eng verwoben sind und die kommunikative Funktion verstärkend auf die kognitive 
wirkt“ (Meyer und Tiedemann 2017, S. 42). Mit Blick auf die Ausbildung im Fach Mathematik ist es daher eine wichtige Aufgabe, die Beziehung der beiden Funktionen sowohl für das Fach als auch für die Planung und Durchführung des eigenen Unterrichts zu erfahren.

Bei der Qualifizierung angehender Mathematiklehrkräfte für einen sprachbildenden Unterricht spielt zunächst die sprachliche Sensibilisierung der Studierenden selbst eine wichtige Rolle. Grundlagenkenntnisse etwa im Bereich der deutschen Grammatik, aber auch zu (beispielsweise typologischen) Unterschieden zwischen Sprachen oder in Bezug auf Verfahren der Sprachdiagnostik, wie sie in Veranstaltungen im Bereich Sprachbildung thematisiert werden, bilden dafür eine wertvolle Basis. Sie sollten in fachdidaktischen Veranstaltungen mit Blick auf die sprachlichen Gegebenheiten des jeweiligen Fachs reflektiert und konkretisiert werden. Dies wird anhand der folgenden Beispiele für das Fach Mathematik veranschaulicht.

\section{- Sprachliche Eigenschaften des Fachs Mathematik}

Die spezifischen Eigenschaften der Sprache der Mathematik bzw. des Schulfachs Mathematik sowie potentielle Schwierigkeitsbereiche für Schüler*innen sind für das Deutsche gut dokumentiert (u. a. Maier und Schweiger 1999; Gürsoy 2016; Prediger 2020; Wessel et al. 2018; Guckelsberger und Schacht 2018 sowie $>$ Abschn. 1.2.1.1). Um die Wahrnehmung der Studierenden für das Thema zu schärfen und ihre analytischen Fähigkeiten zu stärken, bietet es sich an, im Seminar mathematische Texte (z. B. Aufgabenstellungen) aus fachlicher und sprachlicher Perspektive zu untersuchen. Es geht dabei nicht um ein allgemeines sprachanalytisches Training, sondern vor allem um die Fähigkeit, sprachliche Mittel auf ihre fachliche Relevanz hin zu überprüfen: Welche Ausdrücke und Strukturen sind bedeutsam für das fachliche Verstehen? Worauf sollte entsprechend im Unterricht besonders geachtet werden?

Zieht man etwa die Beispiele B1 und B2 vergleichend heran, so wird deutlich, wie eng das Verständnis der fachlichen Sachverhalte im Deutschen mit dem Verständnis der Präpositionen verknüpft ist:

B1-Deutsch - Der Tulpenpreis steigt auf drei Euro.

B2-Deutsch - Der Tulpenpreis steigt um drei Euro.

Die Präpositionen „,auf“ und „um“ stellen ,,auf abstrakter Ebene sprachliche Relationen zu mathematischen Sachverhalten“ (Gürsoy 2016, S. 137) her. Anders als das Verb „,steigen“, das relativ leicht hergeleitet werden kann, falls es Schüler*innen nicht geläufig ist, lässt sich die Bedeutung der Präpositionen ungleich schwerer erfassen: Sie kann nicht aus einem anderen Wort hergeleitet und nur schwer umschrieben werden, auch der Blick ins Wörterbuch hilft kaum weiter. Hinzu kommt, dass die Relationierung von mathematischen Sachverhalten nicht in allen Sprachen über Präpositionen geleistet wird und eine ausdrückliche Thematisierung im Unterricht daher umso wichtiger sein kann. Dies lässt sich im Rahmen einer Lehrveranstaltung leicht verdeutlichen, wenn man auf die sprachlichen Ressourcen der Studierenden zurückgreift. So bestätigte eine Studentin für das Französische eine dem Deutschen vergleichbare Struktur: „Le prix de la tulipe augmente à/de trois Euros [Der Tulpenpreis steigt auf/um drei Euro.]“, während eine andere Studierende für das Finnische feststellte, dass dort die Relationierung nicht über Präpositionen, sondern über unterschiedliche Kasus (Illativ vs. Adessiv) erfolgt. Im Türkischen werden wiederum unterschiedliche Verben verwendet (,yükselmek“"vs. ,,artmak“): 


\begin{tabular}{|c|c|c|c|c|}
\hline B1-Finnisch & $\begin{array}{l}\text { - Tulppaanin hinta } \\
\text { Tulpenpreis }\end{array}$ & $\begin{array}{l}\text { nousee } \\
\text { steigt }\end{array}$ & $\begin{array}{l}\text { kolme-en } \\
\text { drei - Illativ }\end{array}$ & $\begin{array}{l}\text { euro-on. } \\
\text { Euro - Il }\end{array}$ \\
\hline & „Der Tulpenpreis & teigt auf & Euro.“ & \\
\hline 2-Finnisch & $\begin{array}{l}\text { - Tulppaanin hinta } \\
\text { Tulpenpreis } \\
\text { „Der Tulpenpreis }\end{array}$ & $\begin{array}{l}\text { nousee } \\
\text { steigt } \\
\text { teigt um }\end{array}$ & $\begin{array}{l}\text { kolme-lla } \\
\text { drei - Adessiv } \\
\text { Euro.“ }\end{array}$ & $\begin{array}{l}\text { euro-lla. } \\
\text { Euro - A }\end{array}$ \\
\hline B1-Türkisch & $\begin{array}{l}\text { - Lale fiyatı } \\
\text { Tulpenpreis } \\
\text { „Der Tulpenpreis }\end{array}$ & $\begin{array}{l}\text { üç } \\
\text { drei } \\
\text { teigt auf }\end{array}$ & $\begin{array}{l}\text { avro-ya } \\
\text { Euro - Dativ } \\
\text { Euro." }\end{array}$ & $\begin{array}{l}\text { yükseliy } \\
\text { steigt }\end{array}$ \\
\hline 2-Türkisch & $\begin{array}{l}\text { - Lale fiyatı } \\
\text { Tulpenpreis } \\
\text { „Der Tulpenpreis }\end{array}$ & $\begin{array}{l}\text { üç } \\
\text { drei }\end{array}$ & $\begin{array}{l}\text { avro- } \varnothing \\
\text { Euro - Nominativ } \\
\text { Euro.“ }\end{array}$ & $\begin{array}{l}\text { artiyor. } \\
\text { steigt }\end{array}$ \\
\hline
\end{tabular}

Aber auch unterschiedliche Konzeptualisierungen von Fachbegriffen in verschiedenen Sprachen können mit den Studierenden diskutiert werden. So zeigen Prediger et al. (2019a, b) am Beispiel des Deutschen und des Türkischen, wie bei der Konzeptualisierung von Anteilen (z. B. $\frac{3}{5}$ ) im Deutschen vom Teil ausgegangen wird (,drei von fünf“ oder „drei Fünftel“), im Türkischen hingegen vom Ganzen (,,beşte üç“ - „fünf, darin drei“" oder ,beşten üç“" - ,fünf, davon drei“").

Das Nachdenken über mathematisch relevante Phänomene der deutschen Sprache und über den Zusammenhang von Sprachmitteln und Sprachfunktionen in anderen Sprachen erhöht die fachbezogene Sprachbewusstheit der angehenden Lehrkräfte (dies schließt manchmal auch die Erkenntnis ein, dass die Kenntnisse in einer eigentlich gut beherrschten Sprache im Fachkontext an ihre Grenzen kommen). Daran können Überlegungen für gezielte Sprachbildungsmaßnahmen im Mathematikunterricht anknüpfen. ${ }^{19}$

\section{- Lernersprachenanalyse}

Eine hohe fachbezogene Sprachbewusstheit erweist sich auch bei der fachlichen und sprachlichen Analyse von mündlichen und schriftlichen Schülerbeiträgen als nützlich. Dabei geht es in Veranstaltungen im Rahmen der Lehramtsausbildung weniger darum, dass die Studierenden die sprachlichen Fähigkeiten der Lernenden möglichst umfassend einschätzen - dies wäre Aufgabe einer kompetenzorientierten, zum Zweck der Sprachförderdiagnostik durchgeführten Lernertextanalyse (Veiga-Pfeifer et al. 2020). Vielmehr liegt der Fokus wie im vorangegangenen Abschnitt in erster Linie auf Aspekten, die für das fachliche Verstehen bzw. für den Ausbau fachlicher Kommunikationsfähigkeit notwendig sind. Als Grundlage dienen keine allgemeinsprachlichen Schülertexte, sondern mündliche oder schriftliche Äußerungen aus dem Fachunterricht.

- Abb. 1.4 zeigt einen Auszug aus der schriftlichen Aufgabenbearbeitung eines Zehntklässlers, der aus Syrien zugewandert ist und seit zwei Jahren Deutsch lernt. Die Aufgabe erforderte es, die Angaben aus dem Aufgabentext in ein doppeltes Baumdiagramm zu übertragen, diesen Vorgang Schritt für Schritt schriftlich zu beschreiben und

19 So entwickelte ein Studierender ausgehend von der Beobachtung, dass eine Schülerin die Präposition ,,je“ in einem Aufgabentext nicht verstand, sein Studienprojekt, in dem er den Zusammenhang von sprachlichen Anforderungen und Lösungshäufigkeit bei Mathematikaufgaben untersuchte ( Abschn. 3.1). 
- Abb. 1.4 Schülerdokument „Doppeltes Baumdiagramm“ (Auszug)

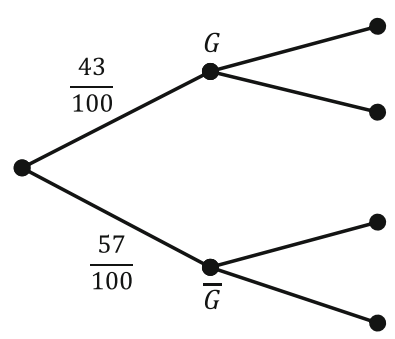

Zuerst der Anzahl die Schüler die von der Grundschule ins Gymnasium gewechselt sind beträgt 43\%. Also $100-43=$ ist $57 \%$ die Anzahl der Schüler die an einen anderen Schulform gewechselt haben.

schließlich Fragen zu bedingten Wahrscheinlichkeiten zu beantworten. Der Auszug dokumentiert den ersten Schritt des Schülers bei der Entwicklung des Baumdiagramms. Inhaltlich geht es um die Wahrscheinlichkeit, dass Kinder aus einem Akademikerhaushalt vs. Nicht-Akademikerhaushalt nach der Grundschule auf das Gymnasium wechseln.

An diesem Beispiel kann diskutiert werden, welche fachlichen und sprachlichen Kompetenzen der Schüler in diesem kleinen Textabschnitt zeigt und welche Rückmeldungen in Bezug auf das Fach Mathematik sinnvoll erscheinen. Dabei ist zunächst festzuhalten, dass der Schüler grundsätzlich in der Lage ist, die Aufgabe zu bearbeiten - dies ist nach nur zwei Jahren Deutsch eine beachtliche Leistung. Fachlich auffällig ist die Verwendung des Ausdrucks „Anzahl“ statt „Anteil“ - dazu sollte eine Rückmeldung und ggf. inhaltliche Klärung (absolute vs. relative Häufigkeit) erfolgen. Im Weiteren kann diskutiert werden, wie man den Schüler bei der Versprachlichung von Konstruktionsprozessen unterstützen und damit seine mathematischen Ausdrucksmöglichkeiten erweitern könnte. Ein Vorschlag wäre etwa eine genredidaktische Herangehensweise, die sprachliche Vorbilder mit einbezieht ${ }^{20}$; dies würde ggf. auch die Grammatikverwendung positiv beeinflussen und ließe sich zudem auf mündliche Kommunikationssituationen (z. B. Versprachlichung von Rechenwegen) übertragen.

\subsection{Leitideen und Design-Prinzipien für eine sprachbewusste Hochschullehre in der Lehramtsausbildung Mathematik}

Im vorliegenden Abschnitt werden zunächst Leitideen zur Verortung von Sprache und Fach in der Lehramtsausbildung entwickelt, die die inhaltlichen Rahmenbedingungen für eine sprachbewusste Hochschullehre markieren und anhand von Beispielen veranschau-

20 z. B. Gibbons (2015), Gürsoy (2018), Jahn (2020). Im Rahmen eines Lehr-Lern-Zyklus wird eine schulisch relevante Fachtextsorte („Genre“) - im Fall von Mathematik z. B. eine Konstruktionsbeschreibung, eine Textaufgabe oder ein Wissensspeicher - im Unterricht schrittweise erarbeitet. Dabei steht die Arbeit am sprachlichen Modell im Mittelpunkt: ein Modelltext wird auf seinen Zweck, seine Struktur und seine sprachlichen Eigenschaften hin untersucht („modeling and deconstructing the genre“); anschließend werden die Lernenden zu einer gemeinsamen Konstruktion und eigenständigen Produktion der Fachtextsorte hingeführt (,,joint construction“, ,,independent writing“). 
lichen ( Abschn. 1.2.1). Vor diesem Hintergrund werden dann hochschuldidaktische Design-Prinzipien für eine fach- und sprachintegrierte sowie forschungsbezogene Hochschullehre in der Lehramtsausbildung Mathematik formuliert, die der praktischen Ausgestaltung der Lehre zugrunde gelegt werden können ( Abschn. 1.2.2). Eine Übersicht über die Leitideen und Design-Prinzipien findet sich in $\rightarrow$ Abschn. 1.2.3.

\subsubsection{Leitideen zur Verortung von Sprache und Fach in der Lehramtsausbildung Mathematik}

Die im Folgenden vorgestellten und an Beispielen entfalteten Leitideen sollen zur Orientierung bei der Planung von Lehrveranstaltungen in der Lehramtsausbildung Mathematik mit einem Schwerpunkt auf Sprachbildung beitragen. Im Kontext einer sprachbewussten Hochschullehre dienen sie insbesondere auch der Einordnung der in $\triangleright$ Kap. 2 diskutierten Konzepte. Ein zentraler Gedanke dabei ist es, für die Studierenden die Möglichkeiten der Vernetzung von Fach und Sprache auf theoretischer und praktischer Ebene in der Hochschullehre (und insbesondere beim Forschenden Lernen) greifbar zu machen und sie auf diese Weise auf den eigenen sprachbildenden Fachunterricht in der Schule vorzubereiten.

Die Leitideen zur Verortung von Sprache und Fach in der Lehramtsausbildung Mathematik beziehen sich auf folgende Bereiche - wobei selbstverständlich kein Anspruch auf Vollständigkeit erhoben werden soll:

L1: Fachbezogenes Sprachwissen als Voraussetzung für sprachbewussten Fachunterricht

L2: Aufgabenkultur

L3: Systematische sprachliche Unterstützung im Fachunterricht

L4: Fachlich-sprachliches Diagnostizieren

Die Grundlage bilden zum einen Kriterien für Sprachbildung im (Fach-)Unterricht und Zielkompetenzbeschreibungen für Lehramtsstudierende bzw. Lehrkräfte im Bereich Sprachbildung, sowie zum anderen entsprechende Bezugstheorien und Forschungserkenntnisse, die die Leitideen aus sprach- und fachdidaktischer Perspektive begründen.

Eine Übersicht über die Leitideen findet sich in $>$ Abschn. 1.2.3.

\subsubsection{L1: Fachbezogenes Sprachwissen als Voraussetzung für sprachbewussten Fachunterricht}

Der reflektierte Umgang mit Sprache im Fachunterricht gilt als wichtiges Mittel, um allen Lernenden den bestmöglichen Zugang zu fachlichen Inhalten zu bieten und das fachliche Verstehen ausgehend von den jeweiligen sprachlichen Fähigkeiten der Lernenden zu fördern (Schmölzer-Eibinger et al. 2013). Dazu gehört eine hohe Aufmerksamkeit für die sprachlichen Anforderungen des jeweiligen Fachs, die auf die fachlichen Anforderungen bezogen und für die Lernenden transparent gemacht werden müssen. Als besonders wichtig wird dabei das In-Beziehung-Setzen von Alltags- und Bildungs-/Fachsprache erachtet (Gogolin et al. 2011): Die von den Lernenden mitgebrachten sprachlichen Ressourcen werden im Unterricht aufgegriffen, fachlich reflektiert und hinsichtlich der kommunikativen, insbesondere auch schriftsprachlichen Anforderungen im Fach sukzessive ausgebaut $(\rightarrow$ Leitidee L3). Bewusster Umgang mit Sprache im Fachunterricht bezieht sich auch auf die herkunfts- und fremdsprachlichen Kenntnisse der Lernenden, die so in den Unterricht 
integriert werden sollen, dass sie zum fachlichen Lernen aller Schülerinnen und Schüler beitragen (Rehbein 2012; Büttner und Gürsoy 2018; Fürstenau und Niedrig 2018).

In Kasten 1 sind eine Reihe von Auszügen aus verschiedenen Leitlinien zur Sprachbildung im Fachunterricht bzw. für die entsprechende Qualifikation von (angehenden) Lehrkräften zusammengestellt:

\section{Kasten 1: Auszüge aus Leitlinien/Empfehlungen für Sprachbildung im} (Fach-)Unterricht bzw. für eine entsprechende Lehrkräftequalifikation

- „Im sprachaufmerksamen Fachunterricht ist die Sprachverwendung durch Sprachaufmerksamkeit und Sprachreflexion geprägt“" (Schmölzer-Eibinger et al. 2013, S. 26).

- „Im sprachaufmerksamen Fachunterricht sind sprachliche Anforderungen explizit und transparent" (Schmölzer-Eibinger et al. 2013, S. 38).

- „Die Lehrkräfte planen und gestalten den Unterricht mit Blick auf das Register Bildungssprache und stellen die Verbindung von Allgemein- und Bildungssprache explizit her" (Gogolin et al. 2011, S. 14).

- „Es herrscht ein bewusster und förderlicher Umgang mit Sprache in allen Fächern und schulischen Handlungsbereichen“ (MSW NRW 2015, 2.7.1., S. 33).

- „Sprachliche Kompetenzen von Schülerinnen und Schülern anderer Herkunftssprachen werden aufgegriffen und berücksichtigt“ (MSW NRW 2015, 2.7.2., S. 34).

- „Wissen über Sprache und Sprachbildung: Wie wird in meinem Fach kommuniziert? Sprache(n) im Fach, Darstellungsformen, morphologische und syntaktische Besonderheiten, BICS und CALP, Schriftlichkeit und Mündlichkeit, Sprachbildung, ... “(Leisen 2016, S. 52).

Damit Studierende als zukünftige Lehrkräfte ihren Fachunterricht sprachbewusst planen und durchführen können, müssen sie sich - über allgemeine linguistische Grundkenntnisse hinaus - spezifisches Wissen hinsichtlich der sprachlichen Eigenschaften ihrer jeweiligen Unterrichtsfächer aneignen. Denn nur dann können sie relevante Anforderungsbereiche und Lernpotentiale identifizieren und produktiv in den Unterricht einbinden. Der Aufbau fachbezogenen Sprachwissens ist also eine zentrale Aufgabe der fach- und sprachintegrierten Hochschullehre.

Für das Fach Mathematik ergibt sich somit folgende Leitidee L1:

L1

Die sprachbewusste Lehramtsausbildung Mathematik sensibilisiert die Studierenden für Sprache im Fach Mathematik und schafft so die Voraussetzung für einen reflektierten Umgang mit Sprache und Mehrsprachigkeit im Mathematikunterricht.

Auf der Unterrichtsebene zeigen empirische Belege, dass ,die Sprachkompetenz unter allen betrachteten sozialen und sprachlichen Faktoren den größten Beitrag zur statistischen Erklärung von Unterschieden in der Mathematikleistung hat" (Prediger et al. 2015, S. 90). Solche empirischen Belege haben wichtige Konsequenzen für die universitären Lerngegenstände: Ein bewusster Umgang mit Sprache im Fach Mathematik ist von zentraler Bedeutung für den schulischen Lernprozess, und dies sollte für die Studierenden erfahrbar sein. Studierende sollten sich als Vorbereitung auf die sprachbewusste Gestaltung 
ihres Unterrichts daher zunächst mit den sprachlichen Eigenschaften des (Schul-)Fachs Mathematik beschäftigen.

\section{L1-1}

Die Studierenden eignen sich spezifisches Wissen in Bezug auf die sprachlichen Eigenschaften des Fachs Mathematik an. Sie identifizieren sprachliche Anforderungen, die für das fachliche Verstehen relevant sind.

Für die Fachsprache der Mathematik liegen detaillierte Beschreibungen vor (z. B. Maier und Schweiger 1999; Meyer und Tiedemann 2017). Bei der Thematisierung in der Lehramtsausbildung Mathematik sollten insbesondere solche Aspekte thematisiert werden, die das fachliche Lernen nachweislich beeinflussen, ohne dass dies auf den ersten Blick erkennbar wäre.

Mathematische Fachbegriffe werden als ,unverzichtbare[r] Bestandteil des Lehrens und Lernens" wahrgenommen (Frank und Gürsoy 2015, S. 136). Während insbesondere Begrifflichkeiten lateinischen oder griechischen, seltener auch englischen Ursprungs in der Regel als typisch mathematisch oder zumindest typisch fachsprachlich erkannt werden (z. B. ,,multiplizieren“, „Logarithmus“, „,Boxplot“), trifft dies auf andere Fachbegriffe nicht in gleichem Maße zu: Ausdrücke wie „Viereck“, ,,abhängig“, ,parallel“, ,,abtragen“ oder „Ereignis“ kommen in modifizierter oder ganz anderer Bedeutung auch in der Alltagssprache vor - es handelt sich also um ,unauffällige Fachwörter" (Guckelsberger und Schacht 2018). So werden beispielsweise unter dem Wort „Viereck“ nach alltagssprachlichem Verständnis zumeist nur Rechtecke oder Quadrate verstanden, wohingegen nach fachlichem Verständnis alle Polygone mit vier Ecken und vier Kanten gemeint sind. Es kann dann - anders als bei Ausdrücken, die ausschließlich im Fachkontext auftreten - zu „Interferenzen zwischen alltagssprachlichen und fachlichen Bedeutungen“ (Maier und Schweiger 1999, S. 121 ff.) kommen. Im Unterricht muss die fachliche Bedeutung sukzessive herausgearbeitet werden. ${ }^{21}$

\section{Beispiel 1}

Die Studierenden identifizieren mathematische Fachbegriffe, die in anderer oder weniger spezifischer Bedeutung auch im Alltag auftreten (,Viereck“, ,abhängig“; ,,parallel“; ,,abtragen“; „Ereignis").

In diesem Zusammenhang ist auch auf fachspezifische Kollokationen, i. d. R. Verbindungen von Substantiven und Verben, hinzuweisen. Sie bezeichnen entweder mathematische Handlungen (z. B. ,,einen Bruch erweitern/kürzen“, ,,eine Gleichung aufstellen/auflösen“, „eine Wurzel ziehen“), bei denen der oder die Mathematiker*in das handelnde Subjekt ist (also z. B. „Ich erweitere den Bruch“ etc.). Oder sie bezeichnen mathematische Zustände (z. B. ,ein Ereignis tritt ein“; ,,die Gerade schneidet [den Kreis]“); ${ }^{22}$ hier liegt die Vorstellung des mathematischen Gegenstands als handelndem Subjekt zugrunde (Maier und Schweiger 1999, S. 22). Auf fachspezifische Kollokationen ist im Unterricht besonders zu achten - zum einen, weil die jeweiligen Bestandteile für sich genommen

21 Vgl. Schacht et al. (i. V.) zur fachspezifischen Ausschärfung des Begriffs „Parallele“ in der Jgst. 6 sowie

- Abschn. 2.4 zum Begriff der „Abhängigkeit“ im Unterrichtsdiskurs der Jgst. 10.

22 Beispiele aus anderen Fächern wären etwa „die Präposition ,[mit]“ verlangt [den Dativ]“ (Fach Deutsch), „die Kraft greift an“ (Fach Physik); s. Tajmel und Hägi-Mead (2017). 
(oft) in anderer Verwendungsweise auch in der Alltagssprache vorkommen, zum anderen, weil sich die Substantive und Verben nicht beliebig variieren lassen. Hinzu kommt, dass die mathematische Bedeutung sich nicht immer leicht erschließen lässt, selbst wenn die Bestandteile für sich genommen bekannt sind (vgl. Tajmel 2011, 2017). Fachspezifische Kollokationen müssen daher im jeweiligen mathematischen Kontext gelernt werden.

\section{Beispiel 2}

Die Studierenden erkennen fachspezifische Kollokationen, die im mathematischen Kontext gelernt werden müssen (z. B. „einen Bruch erweitern“, „,eine Wurzel ziehen“; „,ein Ereignis tritt ein").

Untersuchungen zum mathematischen Aufgabenverständnis offenbaren einen weiteren sprachlichen Anforderungsbereich: Bildungssprachliche Inhaltswörter wie „Auslastung“ oder „Erlös“ sind keine mathematischen Fachwörter; dennoch können sie das mathematische Aufgabenverständnis erheblich beeinflussen (Gürsoy et al. 2013). Anders als Fachtermini sind bildungssprachliche Inhaltswörter kein genuiner Vermittlungsgegenstand des Fachunterrichts und werden entsprechend auch in Merksätzen, Definitionen oder Glossaren nicht berücksichtigt. Umso wichtiger ist es, dass solche Wörter im Unterricht als mögliches Lernhindernis wahrgenommen und bei Bedarf geklärt werden.

\section{Beispiel 3}

Die Studierenden identifizieren in Aufgaben komplexe bildungssprachliche Inhaltswörter wie „Zuschauerschnitt“ oder „Erlös“ (Gürsoy et al. 2013) - d. h. Ausdrücke, die nicht zur Fachsprache der Mathematik im engeren Sinn gehören, jedoch für das Aufgabenverständnis ausschlaggebend sein können. Sie wissen, dass solche Ausdrücke für viele Lernende eine Herausforderung darstellen und ggf. einer expliziten Klärung bedürfen.

Auch Präpositionen zur Relationierung mathematischer Sachverhalte haben, wie Gürsoy et al. 2013 empirisch zeigen, einen erheblichen Einfluss auf das mathematische Aufgabenverständnis ( Abschn. 1.1.3). Sie sind für Lernende schwer zu erfassen, insbesondere wenn in der Erstsprache solche Relationierungen nicht über Präpositionen, sondern über andere sprachliche Mittel (z. B. Kasus, Verben) vollzogen werden. Studierende sollten sich dieser möglichen Problematik bewusst sein und lernen, entsprechende Lerngelegenheiten bereitzustellen (Gürsoy 2016, 198 ff.).

\section{Beispiel 4}

Die Studierenden wissen, dass im Deutschen Präpositionen in abstrahierender Weise genutzt werden, um mathematische Relationen auszudrücken (,Um wie viel Prozent liegt der Verbrauch bei $180 \mathrm{~km} / \mathrm{h}$ über dem Verbrauch bei $100 \mathrm{~km} / \mathrm{h}$ ?“‘23; ,Der Tulpenpreis steigt auf/um $3 €$.“).

Die Sprache des Schulfachs Mathematik zeichnet sich schließlich durch syntaktische Strukturen aus, die vom alltäglichen Gebrauch abweichen. Dazu gehören etwa die für Lernende ungewohnte Positionierung des finiten Verbs an erster (statt zweiter) Position im Satz (,Sei $\varepsilon>0$ vorgegeben.“) oder die Umkehrung der üblichen Wortstellung (Inversion) in Aufgabenstellungen (,Gegeben sei die Funktion $f$... “). Darüber hinaus weisen

23 Item A2a2, Zentrale Prüfungen 10 Mathematik, NRW 2012 (zitiert nach Gürsoy 2016, S. 137). 
Textaufgaben oft komplexe textdeiktische Verweise auf, die für das Aufgabenverständnis erschlossen werden müssen (Gürsoy 2016; Guckelsberger und Schacht 2018).

\section{L1-2}

Die Studierenden entwickeln eine Bewusstheit für die Bedeutung von Mehrsprachigkeit im Fach Mathematik.

Der aufmerksame Umgang mit Sprache im Fachunterricht schließt auch die Berücksichtigung der von den Lernenden gesprochenen Sprachen jenseits des Deutschen, insbesondere von Herkunftssprachen, ein. Studierende sollten wissen, dass mathematische Sachverhalte unterschiedlich konzeptualisiert und versprachlicht werden können und dass sich bspw. aus einer vergleichenden Betrachtung unter Einbezug von Herkunfts- und Fremdsprachen Erkenntnisse für alle Lernenden gewinnen lassen.

\section{Beispiel 1}

Die Studierenden nehmen Sprachvergleiche vor, um zu untersuchen, wie Begriffe, Zahlen oder mathematische Relationen ( Abschn. 1.1.3) in verschiedenen Sprachen konzeptualisiert werden.

\section{Beispiel 2}

Die Studierenden erproben und reflektieren ihre eigenen mehrsprachigen Fähigkeiten (z. B. in Schulfremdsprachen und/oder Herkunftssprachen) im Kontext Mathematik. Sie schärfen ihre Wahrnehmung für die Perspektive von Zweit- oder Fremdsprachenlernenden und für die Nutzung von Erstsprachen als Ressource beim fachlichen Lernen.

Darüber hinaus erweitert die Nutzung von Erstsprachen (und ggf. auch der Brückensprache Englisch; Roth 2018) für viele Schüler*innen die Möglichkeiten zu einer aktiven Teilhabe am mathematischen Diskurs und stellt daher eine wertvolle Ressource für das fachliche Lernen dar (Meyer und Prediger 2011; Meyer und Tiedemann 2017; Redder et al. 2018; Prediger et al. 2019a, b). ${ }^{24}$ Das ist insbesondere für die spezifische sprachliche Situation von neu zugewanderten Lernenden von Bedeutung, die noch nicht über umfassende alltagssprachliche und fachsprachliche Deutschkenntnisse verfügen - unter Umständen aber über gute fachliche Vorkenntnisse (Fürstenau und Niedrig 2018). ${ }^{25}$

Entsprechende Kenntnisse in diesem Bereich sind für Studierende nicht nur für die Schulpraxis relevant, sondern bieten auch interessante Anknüpfungspunkte für forschendes Lernen.

\subsubsection{L2: Aufgabenkultur}

Aufgaben bilden für das Unterrichtsfach Mathematik die „Kristallisationspunkte des selbsttätigen Lernens“ (Neubrand 2002, S. 2). Sie sind zugleich Medium des Lernens

24 Hier sei insbesondere auf das Projekt MuM-Multi 2 - Sprachenbildung im Mathematikunterricht unter Berücksichtigung der Mehrsprachigkeit - verwiesen, in dem Strategien mehrsprachigen Handelns im Fach Mathematik bei Bildungsinländer*innen und neu zugewanderten Schüler*innen untersucht werden.

25 Dies kann zum Beispiel umgesetzt werden, indem die Studierenden eine Mathematikaufgabe in einer selbst gewählten Sprache (Fremd- oder Herkunftssprache) bearbeiten und anschließend aufgetretene Probleme und hilfreiche Strategien besprechen. 
und Anlass zum Lernen. Für die Mathematikdidaktik stellen Aufgaben daher ein reichhaltiges Forschungs- und Entwicklungsfeld dar (für einen Überblick s. z. B. Leuders 2015; Büchter und Leuders 2016; Bruder 2010 oder Shimizu et al. 2010). Insbesondere Beiträge zur fachdidaktischen Entwicklungsforschung verknüpfen Entwicklungsund Forschungsaktivitäten, indem die Aufgabenentwicklung empiriegestützt vorgenommen wird (Hußmann et al. 2011; Gravemeijer und Cobb 2006). In diesem Sinne kann beispielsweise die genaue Beforschung von Lernprozessen von Schülerinnen und Schülern den Ausgangspunkt für die Evaluation entwickelter Aufgaben bzw. komplexerer Lernumgebungen bilden (z. B. Zindel 2019; Schacht 2012).

Die besondere Stellung von Aufgaben im Unterrichtsfach Mathematik spiegelt sich in einer entsprechend intensiven Auseinandersetzung im Mathematikstudium, wo die Konstruktion von Lernumgebungen z. B. im Kontext von Praxisphasen einen wichtigen Schwerpunkt bildet. Gerade weil Aufgaben im Mittelpunkt des Mathematikunterrichts stehen, ist es jedoch von zentraler Bedeutung, dass sich Studierende nicht nur aus fachlicher, sondern auch aus sprachlicher Perspektive mit Aufgaben befassen.

Dass Aufgabenqualität auch ein Thema im Bereich der Sprachbildung ist, zeigen die Qualitätskriterien für Sprachbildung im Fachunterricht bzw. für die Ausbildung von Lehrkräften in diesem Bereich (Kasten 2). Insbesondere die Integration von geeigneten Schreibaufgaben in den Fachunterricht wird als zentral angesehen, da sie zum einen zum Aufbau fach- und bildungssprachlicher Fähigkeiten (kommunikatives Schreiben), zum anderen zur Vertiefung des fachlichen Verstehens (epistemisches Schreiben) beitragen können (Schmölzer-Eibinger et al. 2013; Stephany et al. 2015). Allgemeiner wird gefordert, auf eine sprachsensible Aufgabengestaltung (Leisen 2016) zu achten, den Lernenden umfassende Sprech- und Schreibgelegenheiten anzubieten (MSW NRW 2015, Kriterium 2.7.1) sowie für den sprachsensiblen Fachunterricht geeignete (ggf. Mehrsprachigkeit berücksichtigende) Materialien einzuplanen (ÖSZ 2014).

\section{Kasten 2: Auszüge aus Leitlinien/Empfehlungen für Sprachbildung im}

(Fach-)Unterricht bzw. für eine entsprechende Lehrkräftequalifikation

- „Im sprachaufmerksamen Fachunterricht spielen Schreib- und Textarbeit eine zentrale Rolle“ (Schmölzer-Eibinger et al. 2013, S. 49).

- „Schülerinnen und Schüler erhalten umfassend Sprech- und Schreibgelegenheiten zur Erprobung ihrer Sprachfähigkeiten und entsprechende Orientierungen, wie sie diese weiterentwickeln können“ (MSW NRW 2015, S. 33).

- „Die Studierenden kennen geeignete Materialien für einen sprachsensiblen (Fach-)Unterricht und können diese für ihre Unterrichtsplanung berücksichtigen“ (ÖSZ 2014, Themenbereich 4.9).

- „Die Studierenden kennen die für ihre Schulstufe/n relevanten mehrsprachigkeitsorientierten Materialien und Ressourcen“ (ÖSZ 2014, Themenbereich 5.3).

Allerdings liegen Aufgaben, die fachliches und sprachliches Lernen sinnvoll verknüpfen, nur exemplarisch für ausgewählte Fächer und Themen vor. Die Befähigung der Studierenden, Aufgaben unter Berücksichtigung von Grundsätzen aus Sprachbildung und Fachdidaktik zu variieren und weiterzuentwickeln, ist daher als eine zentrale Aufgabe der fach- und sprachintegrierten Lehramtsausbildung zu sehen. Denn nur wenn die Stu- 
dierenden spezifische sprachliche Schwierigkeitsbereiche ( $\rightarrow$ Leitidee L1) einerseits, das sprachbildende Potential von Aufgaben andererseits erkennen und darüber hinaus in der Lage sind, Aufgaben entsprechend weiterzuentwickeln, können sie die Lernenden fachlich und (fach-)sprachlich angemessen fordern und unterstützen. Dies ist umso wichtiger, als Forschungsbefunde an der Schnittstelle von Fach- und Sprachdidaktik einen deutlichen Zusammenhang zwischen Sprachkompetenz und Mathematikleistung aufzeigen (z. B. Gürsoy et al. 2013). Vor diesem Hintergrund lautet die zweite Leitidee L2:

\section{L2}

Die sprachbewusste Lehramtsausbildung Mathematik befähigt die Studierenden zur Berücksichtigung von Sprache bei der Arbeit mit Aufgaben.

Für eine forschungsbasierte Lehramtsausbildung Mathematik mit einer gemeinsamen Perspektive auf Mathematik und Sprache bietet z. B. die fachdidaktische Entwicklungsforschung einen Bezugspunkt, um die Arbeit an Aufgaben (d. h. deren Analyse, (Weiter-) Entwicklung und Erprobung) zu thematisieren. Im Verlauf dieses Buches werden vielfältige Beispiele aus dem Hochschullalltag thematisiert, bei denen Studierende an Aufgaben arbeiten, diese mit Schülerinnen und Schülern erproben und zugrundeliegende Prozesse (z. B. Bearbeitungsprozesse, Prozesse der Verwendung von Erst- und Zweitsprache oder Besonderheiten von Interaktionsprozessen) genauer beobachten und analysieren.

\section{L2-1}

Die Studierenden bestimmen und überprüfen die sprachlichen Auswirkungen mathematikdidaktisch begründeter Entscheidungen, wenn sie Aufgaben konstruieren und variieren.

\section{Beispiel 1}

Die Studierenden untersuchen die sprachlichen Herausforderungen und/oder Erleichterungen, die sich durch die Nutzung digitaler Werkzeuge ergeben.

\section{Beispiel 2}

Die Studierenden untersuchen die Auswirkungen von Aufgabenvariationen auf die Sprachrezeption (hören, lesen) und Sprachproduktion (sprechen, schreiben).

Eine der grundlegenden Tätigkeiten für die Arbeit mit Aufgaben im Mathematikunterricht besteht darin, dass bereits existierende Aufgaben im Sinne einer stoffdidaktischen Analyse (z. B. Hußmann und Prediger 2016; Winter 1985) zunächst hinsichtlich ihrer fachlichen Struktur und Anforderungen genauer untersucht werden. Für die Hochschullehre bietet die Verknüpfung von inhaltsbezogenen und sprachbezogenen Analysen viel Potential, weil mathematikdidaktische Aspekte (etwa zum fachlichen und sprachlichen Differenzierungspotential von Aufgaben), sprachbezogene Aspekte (etwa zur Rolle von Präpositionen in Aufgaben) sowie mathematisch-stoffdidaktische Aspekte (etwa zur Rolle der zugrunde liegenden begrifflichen Struktur) gemeinsam betrachtet werden können. Bei der Variation von Aufgaben wiederum können die spezifischen Konsequenzen auf den jeweiligen Ebenen verdeutlicht werden. Mathematikdidaktisch begründete Entscheidungen können so hinsichtlich ihrer sprachlichen Auswirkungen reflektiert werden 
(L2-1). Dies kann in der Praxis etwa bedeuten, dass die Variation einer Aufgabe durch Nutzung eines Funktionenplotters sprachlich neue Anforderungen stellt, die für die unterrichtliche Planung entsprechend berücksichtigt werden sollten - gerade weil die Nutzung digitaler Werkzeuge die genutzte Sprache im Mathematikunterricht stark beeinflusst (etwa Hölzl 1996; Weigand 2013; Kaur 2015; Schacht 2015, 2017; Sinclair und Yurita 2008).

\section{L2-2}

Die Studierenden variieren Aufgaben so, dass sowohl mathematische als auch sprachliche Lernprozesse unterstützt werden.

\section{Beispiel 1}

Die Studierenden schaffen durch Aufgabenvariation (z. B. Öffnung von Aufgaben) fachliche Kommunikations- bzw. Argumentationsanlässe.

\section{Beispiel 2}

Die Studierenden entwickeln Aufgaben zur fachbezogenen Sprachreflexion (z. B. Vergleich von Fachbegriffen in Alltags- und Fachsprache; Vergleich von Fachbegriffen oder Konzepten in verschiedenen Sprachen).

Ein zentrales Thema bildet die Variation von Aufgaben (z. B. Schupp 2002 oder auch Büchter und Leuders 2016). Unterschiedliche Strategien der Aufgabenvariation wie z. B. das Weglassen von Informationen, die Änderung von Bedingungen oder auch die Verallgemeinerung von Aufgaben tragen zu einer verbesserten mathematischen Aufgabenkultur bei, indem die veränderten Aufgaben nun z. B. das Einbringen von Vor- und Alltagswissen ermöglichen, Kooperation unter den Lernenden fördern, Anlässe zum Argumentieren bieten oder zur Reflexion über Problemlösestrategien anregen (s. vom Hofe 2001; Feindt 2010; Leuders et al. 2011; Büchter und Leuders 2016). Solche aus (primär) mathematikdidaktischen Überlegungen heraus veränderten Aufgaben haben häufig zugleich ein hohes sprachbildendes Potential, weil sie produktives, kooperatives und reflexives Handeln erfordern - Aspekte, die auch in Leitlinien für Sprachbildung im Fachunterricht eine wichtige Rolle spielen.

Für den vorliegenden Kontext werden solche Aufgabenvariationen im Lichte des sprachbewussten Umgangs mit Aufgaben in der Hochschullehre thematisiert ( Abschn. 2.2). Studierende sollten bspw. Aufgaben so variieren können, dass sowohl sprachliche als auch fachliche Lernprozesse unterstützt werden. Die Variation von Aufgabenserien hin zu operativ strukturierten Aufgaben (z. B. Wittmann 1992) kann beispielsweise Anlässe schaffen, um zugrundeliegende mathematische Muster zu beschreiben und zu begründen. Auch der Vergleich unterschiedlicher Zugänge, die durch verschiedene Aufgaben vorgenommen werden (müssen), kann - fachliche ebenso wie sprachliche - Reflexionsprozesse unterstützen.

\section{L2-3}

Die Studierenden entwickeln Schreibaufgaben, die das mathematische Lernen unterstützen. 


\section{Beispiel 1}

Die Studierenden entwickeln Schreibaufgaben zur Vernetzung unterschiedlicher Darstellungsformen.

\section{Beispiel 2}

Die Studierenden entwickeln Schreibaufgaben, die der Systematisierung und Vertiefung des Gelernten dienen (z. B. (mehrsprachiger) Wissensspeicher; Konstruktionsbeschreibung).

Ein in diesem Zusammenhang besonders hervorzuhebender Aspekt ist die Schaffung von Schreibanlässen (etwa Kuntze und Prediger 2005; Götze 2013; Stephany, Linnemann und Wrobbel 2015). Um schriftsprachliche Kompetenzen im Mathematikunterricht auszubauen und das fachliche Verstehen zu vertiefen, können Schreibaufgaben unterschiedlicher Komplexität initiiert werden - etwa zur Darstellungsvernetzung ${ }^{26}$ (Beschriftung einer Graphik; Vergleich von Darstellungsformen), im Kontext einer Entdeckungsphase (Vorhersagen schriftlich festhalten und mathematisch überprüfen (Schacht et al. i. V.); fachliches Vorwissen aktivieren ${ }^{27}$ ) oder zur Wissenssicherung (Ergebnissicherung in SMSoder Briefform ${ }^{28}$ ).

In Kap. 2 wird deutlich, welch zentralen Stellenwert die Entwicklung und Konstruktion von Aufgaben für die Ausbildung zukünftiger Mathematiklehrkräfte hat und welches Potential sich dabei nicht zuletzt im Kontext des forschenden Lernens eröffnet.

\subsubsection{L3: Systematische sprachliche Unterstützung im Fachunterricht}

॥V Verbesserter Zugang zur Mathematik, nicht Sprachförderung per se, sollte das primäre Ziel sein, an dem sich Maßnahmen zur Sprachförderung im Mathematikunterricht zunächst einmal messen lassen müssen (Meyer und Prediger 2011, S. 199).

Sprache ist im Unterricht zugleich Lernmedium (Lernen ist ganz überwiegend sprachlich vermittelt) und Lerngegenstand (fachliche Kommunikationsweisen müssen erlernt werden). Es gehört daher zu den zentralen Fragen des sprachbildenden Fachunterrichts, wie Lernende bei der Aneignung von Fachinhalten sowie, eng damit verknüpft, beim Aufbau fachbezogener Kommunikationsfähigkeiten systematisch sprachlich unterstützt werden können. Grundlage ist eine Bedarfsanalyse, die die sprachlichen und fachlichen Anforderungen des jeweiligen Inhaltsbereichs mit den jeweiligen Voraussetzungen bei den Lernenden abgleicht.

Ansätze zur sprachlichen Unterstützung im Fachunterricht lassen sich grob unterteilen in planbare und nicht (im Detail) planbare Unterstützungsmaßnahmen (Hammond und Gibbons 2005 zur Untergliederung nach Macro-Scaffolding auf Planungsebene und Micro-Scaffolding auf interaktionaler Ebene). Planbar ist beispielsweise eine Phasierung des Unterrichts, bei der Zusammenhänge von sprachlichen und fachlichen Lerngelegenheiten unter Berücksichtigung der jeweiligen fachdidaktischen Grundsätze sichtbar werden. In $\triangleright$ Abschn. 1.1.1 wurde dies am Beispiel des Mathematikunterrichts gezeigt: Prinzipien des Macro-Scaffoldings (Hammond und Gibbons 2005; Gibbons 2006, 2015;

26 Sprachbildung durch Vernetzung von Darstellungsformen wird in $>$ Abschn. 2.3 diskutiert.

27 Vgl. die studentische Erhebung zum Wahrscheinlichkeitsbegriff ( Abschn. 2.6).

28 Vgl. die studentische Erhebung zum Thema Mittelwerte ( $\bullet$ Abschn. 2.2). 
Kniffka 2010) wurden in Beziehung gesetzt zu Kernprozessen mathematischen Lernens (Erkunden, Systematisieren, Vertiefen; Prediger et al. 2014).

Ebenfalls planbar sind bestimmte Arbeits- und Sozialformen, die eine sprachliche Entlastung bewirken (z. B. think - pair - share; Bildung von Arbeitsgruppen mit gemeinsamen Sprachkenntnissen ${ }^{29}$ ) sowie die fach- und sprachbewusste Variation von Aufgaben und weiteren Lernmaterialien, ggf. auch unter gezielter Einbeziehung von Herkunfts- und Fremdsprachen ( $\rightarrow$ Leitidee L2).

Auf andere Arten der Unterstützung können sich (angehende) Lehrkräfte zwar grundsätzlich vorbereiten ${ }^{30}$, sie können sie jedoch nicht im Detail vorausplanen. So gehört zu den zentralen Kriterien sprachbewussten Fachunterrichts die Umsetzung von MicroScaffolding (Hammond und Gibbons 2005), d. h. spontaner Unterstützungsangebote in der mündlichen Interaktion. Während manche Aspekte (z. B. Zeit-Geben bei Schülerantworten) relativ problemlos umsetzbar erscheinen, erfordern andere Unterstützungsleistungen - etwa das Aufgreifen und fachlich adäquate Umformulieren von alltagssprachlichen Äußerungen, ein angemessener Umgang mit sprachlichen Fehlern oder auch die eigene sprachliche Vorbildhaftigkeit - ein hohes Maß an Sprachwissen und sprachlicher Aufmerksamkeit ( $\rightarrow$ Leitidee L1) seitens der Lehrperson.

In Kasten 3 ist eine Auswahl entsprechender Empfehlungen und Leitlinien für Sprachbildung im Fachunterricht bzw. für die entsprechende Qualifizierung von Lehrenden aufgeführt.

\section{Kasten 3: Auszüge aus Leitlinien/Empfehlungen für Sprachbildung im} (Fach-)Unterricht bzw. für eine entsprechende Lehrkräftequalifikation

- „Im sprachaufmerksamen Fachunterricht erfolgt eine systematische sprachliche Unterstützung“ (Schmölzer-Eibinger et al. 2013).

- „Die Studierenden kennen Strategien des sprachsensiblen (Fach-)Unterrichts und können diese für ihre Unterrichtsplanung berücksichtigen“ (ÖSZ 2014, Themenbereich 4.10).

- „Die Sprachstände der Schülerinnen und Schüler werden bei der Planung und Gestaltung der unterrichtlichen Prozesse mit dem Ziel berïcksichtigt, fachliche Verstehensprozesse zu erleichtern und bildungssprachliche Kompetenzen aktiv zu fördern“ (MSW NRW 2015, S. 33).

- „Die herkunftssprachlichen Hintergründe der Schülerinnen und Schüler werden bei der Planung und Gestaltung des Unterrichts berïcksichtigt“ (MSW NRW 2015, S. 34).

- „Die Lehrkräfte stellen allgemein- und bildungssprachliche Mittel bereit und modellieren diese" (Gogolin et al. 2011, S. 18).

— „Die Lehrkräfte sind Sprachvorbild“ (MSW NRW 2015, S. 33).

29 Einblicke in „Praktiken der Mehrsprachigkeit“ in einer Klasse mit neuzugewanderten Lernenden, die über vielfältige Sprachkenntnisse verfügen und sich dadurch gegenseitig beim Lernen unterstützen können, geben Fürstenau und Niedrig (2018).

30 Eine Vorbereitung kann z. B. durch die Arbeit mit Videos oder Transkripten erfolgen, an denen lehrerseitiges sprachliches Handeln untersucht und ggf. Alternativen gesucht werden. Im Rahmen von Aktionsforschung kann auch das eigene sprachliche Handeln in Lehr-Lernsituationen analysiert und systematisch verändert werden. 
Für die Lehramtsausbildung Mathematik ergibt sich die folgende Leitidee L3:

L3

Die sprachbewusste Lehramtsausbildung Mathematik befähigt die Studierenden, die Schüler*innen beim fachlichen Lernen sowie beim Ausbau von mathematisch relevanten Kommunikationsfähigkeiten systematisch zu unterstützen.

Dabei geht es zunächst um solche Unterstützungsleistungen, die in der Vorbereitung von Lernprozessen im Mathematikunterricht berücksichtigt werden können, also planbar sind:

\section{L3-1}

Die Studierenden berücksichtigen das Thema Sprache bei der Planung von Mathematikunterricht.

Für die Planung einer sprachbildenden Unterrichtseinheit ist es notwendig, den jeweiligen mathematischen Inhaltsbereich (z. B. negative Zahlen; stochastische Abhängigkeit; bedingte Wahrscheinlichkeiten) vorab nicht nur stoffdidaktisch, sondern auch sprachlich zu analysieren ( $\rightarrow$ Leitidee L1). Die Studierenden sollten in diesem Zusammenhang lernen, für die fachliche Auseinandersetzung zentrale Begriffe und Strukturen herauszuarbeiten und ggf. eine systematische Vermittlung vorzubereiten. Das schließt auch die Analyse der für die Unterrichtseinheit vorgesehenen Aufgaben ${ }^{31}$ und weiteren Lernmaterialien ein.

\section{Beispiel 1}

Die Studierenden überprüfen die sprachlichen Anforderungen des Inhaltsbereichs und wählen zentrale Begriffe und Strukturen der Fach- bzw. Bildungssprache zur Thematisierung im Unterricht aus (im Inhaltsbereich „,bedingte Wahrscheinlichkeiten“ z. B. „,bedingt“, ,wenn man weiß, dass ...").

Bei der Planung selbst sollten die Studierenden eine Sensibilität dafür entwickeln, welche sprachlichen Potentiale die unterschiedlichen Phasen des Unterrichts jeweils bieten. So ist es beispielsweise für den Lernprozess von Bedeutung, in der Systematisierungsphase nicht nur das fachliche Wissen zu sichern, sondern zugleich den Übergang von der Alltags- zur Fachsprache zu vollziehen. Dies kann durch bestimmte Aufgabenformate gezielt unterstützt werden.

\section{Beispiel 2}

Die Studierenden beziehen sprachliche Aktivitäten sinnvoll auf mathematische Kernprozesse (z. B. Erstellen eines Wissensspeichers in der Systematisierungsphase; kooperatives Schreiben eines argumentativen Texts in der Vertiefungsphase). ${ }^{32}$

Bei der Planung von Unterricht sollten die Studierenden zudem die Bedingungen mündlicher und schriftlicher Kommunikationssituationen vor Augen haben.

31 Vgl. Leitidee L2 zur zentralen Rolle von Aufgaben im Mathematikunterricht.

32 Vgl. Abschn. 1.1.1. 
Die Studierenden berücksichtigen bei der Unterrichtsplanung Herausforderungen und Potentiale mündlicher und schriftlicher Kommunikationssituationen.

Mündliche face-to-face-Situationen erfordern spontanes sprachliches Handeln. Sie können daher einerseits Lernende unter Druck setzen, sowohl bei der Rezeption (z. B. Verstehen einer lehrerseitigen Erklärung) als auch bei der Produktion (z. B. mündliche Erläuterung des eigenen Rechenwegs). Andererseits lässt sich mit elementaren sprachlichen und nicht-sprachlichen Mitteln v. a. mit Hilfe von Darstellungen eine schnelle und i. d. R. auch eindeutige Verständigung zwischen den Kommunikationspartnern herstellen, was gerade für Lernende mit geringen (Fach-)Deutschkenntnissen eine erhebliche Entlastung bietet.

Auch schriftliche Kommunikationssituationen können zunächst eine große Herausforderung darstellen. So fällt es beispielsweise vielen Lernenden schwer, in Experimentiersituationen auf der Grundlage eigener Beobachtungen Hypothesen schriftlich aufzustellen und mathematisch zu begründen. Auf der anderen Seite können schriftliche Kommunikationssituationen aber entlastend wirken: Die Lernenden haben die Möglichkeit, sich intensiv mit ihrem eigenen Text (beim Schreiben) oder mit einem fremden Text (beim Lesen) zu befassen und dabei ohne Zeitdruck auf sprachliche Unterstützung (z. B. sprachliche Vorbilder; Wörterbücher; Kooperation mit anderen Lernenden) zurückzugreifen.

Studierende sollten also bei der Planung von Unterricht zum einen das jeweilige entlastende Potential von Mündlichkeit und Schriftlichkeit nutzen, zum anderen für herausfordernde Situationen Unterstützungsangebote vorsehen.

Leitidee L3-2 spezifiziert die vorangegangene Leitidee L3-1 mit Blick auf die Nutzung mehrsprachiger Ressourcen:

\section{L3-2}

Die Studierenden entwickeln mathematische Lernumgebungen, die die Mehrsprachigkeit der Lernenden explizit adressieren.

Viele mehrsprachige Sprechende sind in der Lage, je nach Situation flexibel auf die ihnen zur Verfügung stehenden Sprachen zurückzugreifen. Die Nutzung des gesamtsprachigen Repertoires stellt für sie also den Normalfall dar und dient der optimalen Verständigung. ${ }^{33}$ An deutschen Schulen wird als Sprache des Unterrichts allerdings häufig nur das Deutsche akzeptiert, auch wenn Forschungen das Potential mehrsprachiger Lernkontexte belegen (für das Fach Mathematik z. B. Moschkovich 2005; Redder et al. 2018; Prediger et al. 2019a, b). Studierende als angehende Lehrkräfte sollten sich daher mit der Frage auseinandersetzen, wie das fachliche Lernen durch den Einbezug von Herkunfts- und Schulfremdsprachen im Unterricht unterstützt werden kann. So kann etwa die Ermöglichung mehrsprachiger Einzel-, Partner- und Gruppenarbeit eine wichtige Erweiterung der Interaktionsmöglichkeiten darstellen, was besonders für die Gruppe neuzugewanderter Schüler*innen u. U. entscheidend zum fachlichen Verstehen beiträgt (Fürstenau und Niedrig 2018; Roth 2018). Gezielte Sprachvergleiche begrifflicher oder konzeptueller Natur bieten allen Schüler*innen die Möglichkeit zur vertieften Auseinandersetzung

33 Vgl. zur Rolle von Denk- und Arbeitssprache Grießhaber et al. (1996), Rehbein (2011), Rehbein und Çelikkol (2018) sowie zum Konzept des Translanguaging z. B. García und Wei (2015), Gantefort und Maahs (2020). 
mit den Lerngegenständen (z. B. Aufbau des Zahlsystems; Bruchrechnung; Begriff der Wahrscheinlichkeit im Sprachvergleich; Meyer und Tiedemann 2017).

\section{Beispiel 1}

Die Studierenden planen (z. B. in Erkundungsphasen) mehrsprachige Partner- oder Gruppenarbeiten ein.

\section{Beispiel 2}

Die Studierenden planen Sprachvergleiche ein, die allen Lernenden eine vertiefte sprachliche und inhaltliche Auseinandersetzung mit fachlichen Inhalten ermöglichen.

Neben den in L3-1 und L3-2 ausgeführten Aspekten der planbaren Unterstützung sollten die Studierenden aber auch Möglichkeiten der spontanen Unterstützung im Unterrichtsdiskurs kennenlernen:

\section{L3-3}

Die Studierenden lernen Möglichkeiten kennen, wie sie Lernende in mündlichen LehrLern-Kontexten spontan in mathematischer und sprachlicher Hinsicht unterstützen können.

Die Studierenden sollten lernen, in der direkten Interaktion mit Lernenden verstehensorientiert (vgl. Prediger 2013) zu handeln und Grundsätze des Micro-Scaffoldings zu berücksichtigen. Im Kontext des Mathematikunterrichts heißt das, dass sie mündliche Beiträge von Lernenden - wie die Erläuterung eines Rechenwegs oder die Verbalisierung von Beobachtungen beim mathematischen Experimentieren - würdigend aufgreifen und fachlich wie sprachlich weiterentwickeln. Bei sprachlichen Unsicherheiten ist darauf zu achten, dass die Rückmeldung primär zu solchen Aspekten erfolgt, die für das fachliche Lernen und den Ausbau fachlicher Kommunikationsfähigkeiten relevant sind. Dazu gehört insbesondere die Fähigkeit, Lernendenäußerungen zu erkennen und aufzugreifen, die auf unabgeschlossene fachliche Verstehensprozesse (etwa bei der Begriffsbildung) hinweisen. ${ }^{34}$

\section{Beispiel 1}

Die Studierenden handeln verstehensorientiert und berücksichtigen Grundsätze des MicroScaffolding (z. B. Zeit geben, aktiv zuhören, zusammenfassen).

\section{Beispiel 2}

Die Studierenden reagieren angemessen auf fachliche und/oder sprachliche Unsicherheiten in Lernendenäußerungen.

Praxisphasen im Studium bieten eine gute Gelegenheit, spontanes sprachförderliches Handeln zu beobachten, zu erproben und zu reflektieren, weil die Studierenden im Unterricht oder im Rahmen von Diagnose- und Fördersitzungen in direkten Kontakt mit Lernenden treten. Zur Vorbereitung kann in universitären Lehrveranstaltungen mit Audio-/ Videoaufnahmen oder Transkripten aus mathematischen Lehr-Lern-Kontexten gearbeitet werden ( Abschn. 2.4 und 2.5).

34 Vgl. das Transkriptbeispiel in Abschn. 1.1.1 sowie Schacht et al. (i. V.). 


\subsubsection{L4: Fachlich-sprachliches Diagnostizieren}

Das Diagnostizieren gehört zu den essentiellen Tätigkeiten im schulischen Alltag von Lehrpersonen. Forderungen nach einer Stärkung der Diagnosekompetenz haben ihre Ursprünge nicht zuletzt in den großen Leistungsdefiziten bei Vergleichsstudien wie PISA und TIMSS der 1990er Jahre; durch eine gezieltere Diagnose und Förderung versprach man sich eine Verbesserung der Ergebnisse. Auch vor diesem Hintergrund sind die langjährigen gleichermaßen wissenschaftlich (z. B. DMV, GDM, und MNU 2008) wie bildungspolitisch (z. B. KMK 2003) begründeten Bestrebungen zur Verbesserung der Diagnosekompetenz bei Lehrkräften zu verstehen. So sind etwa für das Fach Mathematik diagnostische Kompetenzen (z. B. Lernausgangsdiagnose) in den Empfehlungen von DMV, GDM und MNU (2008) als unterrichtsbezogene Handlungskompetenzen explizit ausgewiesen (für einen Überblick der mathematikdidaktischen Diskussion zum Thema Diagnostizieren s. etwa Moser Opitz und Nührenbörger 2015).

Aus eher allgemeinpädagogischer Sicht beschreibt Helmke (2009) Diagnose folgendermaßen: „Dabei handelt es sich um ein Bündel von Fähigkeiten, um den Kenntnisstand, die Lernfortschritte und die Leistungsprobleme der einzelnen Schüler sowie die Schwierigkeiten verschiedener Lernaufgaben im Unterricht fortlaufend beurteilen zu können, so dass das didaktische Handeln auf diagnostische Einsichten aufgebaut werden kann." (Helmke 2009, S. 121) In diesem Zusammenhang betonen Moser Opitz und Nührenbörger (2015) die Bedeutung unterschiedlicher Diagnosebegriffe: Im Unterschied zu einer eher lernstandsorientierten Testdiagnostik (abschätzig bisweilen auch als selektionsorientierte Testdiagnostik bezeichnet) orientiert sich eine eher lernprozessorientierte Förderdiagnostik daran, welche Art der „Förderung aus den Beobachtungen abgeleitet werden kann bzw. dass die Beobachtungen Hinweise für Fördermaßnahmen geben“ (Moser Opitz und Nührenbörger 2015, S. 493-494). Aus fachdidaktischer Perspektive geht es insofern beim Diagnostizieren ,um die fachdidaktisch orientierte Erfassung und Analyse der mathematischen Kompetenzen und Lernprozesse einer Schülerin oder eines Schülers.“ (Moser Opitz und Nührenbörger 2015, S. 508).

In einer solchen förderorientierten Perspektive schließt die Diagnose demnach die Reflexion über mögliche Konsequenzen im Rahmen entsprechender Fördermaßnahmen explizit mit ein. Allerdings: „Fördermaßnahmen lassen sich nicht aus den Diagnosen ableiten, sondern aus fachlichen und fachdidaktischen Grundlagen sowie theoretischen Konzepten zur Entwicklung mathematischer Kompetenzen. Nur auf einer solchen Grundlage können Diagnosen geplant und durchgeführt, deren Ergebnisse analysiert sowie Fördermaßnahmen geplant und evaluiert werden - Fördermaßnahmen, die individuelle Lernkompetenzen stärken, mathematische Lösungsprozesse und nicht allein einzelne Produkte thematisieren und den Blick auf die Verringerung von Lernschwierigkeiten richten." (Moser Opitz und Nührenbörger 2015, S. 508) Das hier angesprochene fachliche und fachdidaktische Wissen als Grundlage für die Entwicklung entsprechender Förderungen ist für das unterrichtliche Handeln daher von entscheidender Bedeutung. Für den Mathematikunterricht ergeben sich Diagnoseanforderungen auf unterschiedlichen Ebenen. So lassen sich aus inhaltlicher Sicht etwa Vorstellungen, Verfahren, Begriffsbildungen oder Modellierungskompetenzen mit jeweils spezifischen (Aufgaben-)Formaten diagnostizieren (Hußmann et al. 2007; Bell 1983; Prediger 2006; Reiff 2006).

Diagnose und Förderung fachlicher Kompetenzen bilden dementsprechend auch feste Bestandteile der universitären Lehramtsausbildung. Im Rahmen fachdidaktischer Lehr- 
veranstaltungen steht etwa der Kompetenzerwerb im Umgang mit Heterogenität und Inklusion im Mittelpunkt, insbesondere die Aneignung theoretischer Grundlagen zur Diagnose (insbesondere auch zur Lernprozessdiagnose) und entsprechender Kenntnisse für eine schüler*innengerechte Rückmeldung, Beratung und Förderung.

Um den Fachunterricht auf die individuellen sprachlichen Voraussetzungen der Lernenden auszurichten und so das fachliche und (fach-/bildungs-)sprachliche Lernen gezielt zu unterstützen oder überhaupt erst zu ermöglichen, sollten Fachlehrkräfte auch über Kenntnisse im Bereich der Sprachdiagnostik verfügen. Dafür muss - bezogen auf das Fach Mathematik - die auf mathematische Fähigkeiten und Fertigkeiten ausgerichtete Diagnosekompetenz um eine sprachliche Dimension erweitert werden.

Die Grundlage dafür bieten Leitlinien zur Sprachbildung im Fachunterricht (Kasten 4), die jedoch wiederum hinsichtlich der Erfordernisse des Fachs Mathematik spezifiziert werden müssen.

\section{Kasten 4: Auszüge aus Leitlinien/Empfehlungen für Sprachbildung im} (Fach-)Unterricht bzw. für eine entsprechende Lehrkräftequalifikation

- „Die Lehrkräfte diagnostizieren die individuellen sprachlichen Voraussetzungen und Entwicklungsprozesse“ (Gogolin et al. 2011, Q2).

- „Die Sprachstände der Schülerinnen und Schüler werden bei der Planung und Gestaltung der unterrichtlichen Prozesse mit dem Ziel berücksichtigt, fachliche Verstehensprozesse zu erleichtern und bildungssprachliche Kompetenzen aktiv zu fördern“ (MSW NRW 2015, S. 33).

- „Die Studierenden kennen einige grundlegende Fachbegriffe der Sprachdiagnostik und können den Wert und Nutzen von Sprachstandsbeobachtungen für den eigenen Unterricht erkennen und Förderprozesse initiieren“ (ÖSZ 2014, S. 15).

- „Die Studierenden kennen die Bedeutung von Fehlern und können sie adäquat einschätzen und evaluieren“ (ÖSZ 2014, S. 15).

Im Fokus einer sprachbewussten Lehramtsausbildung Mathematik steht somit die Gewinnung solcher sprachdiagnostischen Erkenntnisse, die für das fachliche und sprachliche Lernen im Fach Mathematik weiterführend sind. Daraus ergibt sich folgende Leitidee L4:

Die sprachbewusste Lehramtsausbildung Mathematik befähigt die Studierenden, bei der Diagnose von Lernendenbeiträgen fachliche und sprachliche Aspekte aufeinander zu beziehen und entsprechende Konsequenzen für das unterrichtliche Handeln im Sinne eines sprachbildenden Fachunterrichts abzuleiten.

Leitidee L4 umfasst zwei Schwerpunkte, die selbstverständlich eng miteinander verknüpft sind: L4-1 bezieht sich auf das fachlich-sprachliche Diagnostizieren mit dem Ziel, das mathematische Verständnis der Lernenden einzuschätzen und zu fördern, während L4-2 die Einschätzung und Förderung mathematischer Kommunikationsfähigkeit in den Vordergrund stellt (vgl. - Abb. 1.5). 


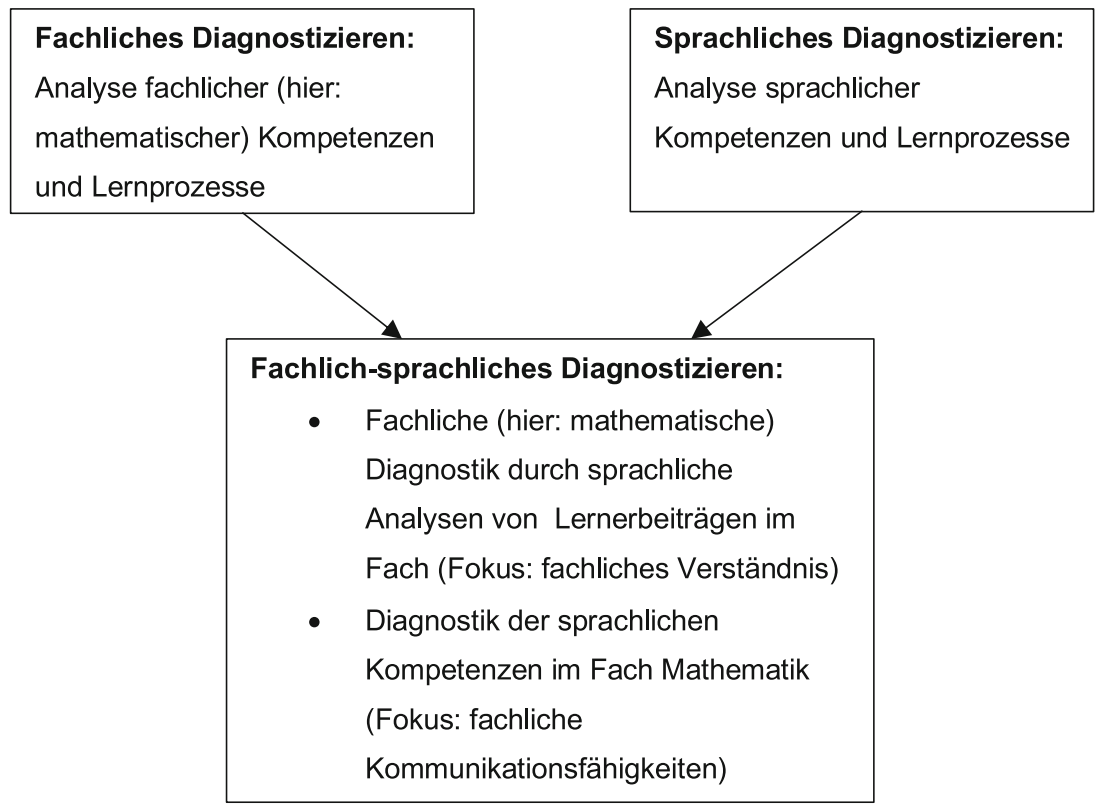

- Abb. 1.5 Fachlich-sprachliches Diagnostizieren

\section{L4-1}

Die Studierenden nutzen mündliche und schriftliche Lernendenbeiträge zur Diagnose des mathematischen Wissens und Könnens und leiten daraus entsprechende fachdidaktisch, fachlich und sprachlich begründete Fördermaßnahmen ab.

Leitidee L4-1 stellt das mathematische Diagnoseinteresse im engeren Sinn in den Mittelpunkt: Es geht um die Frage, welche Auskünfte Formulierungen, Gesten oder Handlungen von Lernenden über die mathematischen Fähigkeiten und Fertigkeiten geben und welche fachdidaktisch, fachlich und sprachlich begründeten Fördermaßnahmen sich daraus ableiten lassen (Frank und Gürsoy 2014; Prediger 2020).

\section{Beispiel 1}

Die Studierenden nutzen Lernendenäußerungen für die Diagnose individueller Vorstellungen, die den Äußerungen zugrunde liegen (z. B. „Wahrscheinlichkeit: was fast perfekt ist in \%.“; „Der Graph wird schmaler.“).

\section{Beispiel 2}

Die Studierenden erkennen und problematisieren Formulierungen, die auf unabgeschlossene mathematische Begriffsbildungsprozesse hinweisen (z. B. „Die Geraden sind ein bisschen parallel. $\left.{ }^{* 35}\right)$. 
Für die Praxis der Hochschullehre heißt das etwa, dass Studierende Lernendenäußerungen hinsichtlich fachlicher Konzeptbildungen diagnostizieren und vor diesem Hintergrund Maßnahmen zur Unterstützung individueller Begriffsbildungen diskutieren. Um die Aufmerksamkeit für das diagnostische Potential spontansprachlicher Äußerungen in Lehr-Lern-Kontexten zu erhöhen und mögliche lehrerseitige Reaktionen durchzuspielen, können Video- oder Audiosequenzen (ggf. als Transkript) genutzt werden.

Studierende sollten zudem Möglichkeiten für die Entwicklung von Aufgaben kennenlernen, aus deren Beantwortung sich Rückschlüsse auf das fachliche Verstehen ziehen lassen. Hierfür eignen sich insbesondere Aufgaben zum mathematischen Argumentieren, denn: „Argumentieren bedarf der Erläuterung. Erläutern ist eine für den Lehr-LernProzess sehr bedeutsame Aktivität: Erläutern deckt Verborgenes auf, Erläutern macht Unsichtbares sichtbar, Erläutern liefert diagnostische Auskünfte.“ (Sjuts 2007, S. 35) Der Kontext des forschenden Lernens (z. B. im Praxissemester) bietet den Studierenden vielfältige Anknüpfungspunkte, um mit solchen Aufgabenformaten zu experimentieren etwa indem sie Schüler*innen zur Versprachlichung von Vorwissen, Lösungswegen oder Ergebnissen anregen oder verstehensorientierte Aufgaben entwickeln, die Begründungen, Interpretationen, Darstellungswechsel und Reflexionen verlangen (vgl. $>$ Abschn. 2.5).

Leitidee L4-2 fokussiert demgegenüber die Diagnose und Förderung der (fach-) sprachlichen Fähigkeiten von Lernenden im Kontext Mathematik:

\section{L4-2}

Die Studierenden analysieren mündliche und schriftliche Lernendenbeiträge und leiten daraus ab, über welche mathematisch relevanten sprachlichen Fähigkeiten die Lernenden schon bzw. noch nicht verfügen. Sie bestimmen den fach- und bildungssprachlichen Unterstützungsbedarf.

Ziel ist es, die fach- und bildungssprachliche Kompetenz der Lernenden auszubauen und ihnen die Teilhabe am mathematischen Diskurs zu ermöglichen. Dabei geht es zum einen um die Diagnose rezeptiver Fähigkeiten: Studierende sollten beispielsweise erkennen, wenn Lernende mathematische Textaufgaben nicht bearbeiten können, weil ihnen bestimmte Begriffe fehlen (L4-2, Beispiel 1) oder weil sie komplexe Verweisstrukturen nicht nachvollziehen können (Gürsoy 2016; Guckelsberger und Schacht 2018). Hierfür ist eine gute Kenntnis der sprachlichen Eigenschaften des Schulfachs Mathematik notwendig $(\rightarrow$ Leitidee L1).

\section{Beispiel 1}

Die Studierenden stellen bei der Analyse eines Schülerinterviews fest, dass der bildungssprachliche Ausdruck „Erlös“ in einer mathematischen Textaufgabe nicht als „Gewinn“ verstanden wird, sondern im Sinn von ,,jemand wird von etwas erlöst“ (und folgerichtig als ,,jemandem wird etwas weggenommen“). ${ }^{36}$

Zum anderen geht es darum, die produktiven kommunikativen Fähigkeiten der Lernenden zu stärken, d. h. ihre mündlichen und schriftlichen Ausdrucksmöglichkeiten im Fach Mathematik zu erweitern; Voraussetzung dafür ist die sprachliche Diagnose der von den Lernenden eingebrachten sprachlichen Mittel (L4-2, Beispiel 2).

36 Vgl. das Transkriptbeispiel in Gürsoy et al. (2013, S. 7). 
Die Studierenden stellen fest, dass die Lernenden bei schriftlichen Interpretationen von Schaubildern mathematische Fachbegriffe zur Beschreibung der Darstellung verwenden (,Diagramm“, „X-Achse“), dass sie aber nicht über den fachlich angemessenen Wortschatz zur Verbalisierung des linearen Wachstums verfügen (z. B. „steigen + um/auf“, ,,je ... desto“).

Den Studierenden muss dafür zunächst selbst bewusst werden, welche Struktur und welche sprachlichen Mittel beispielsweise eine mathematische Textsorte wie die Diagramminterpretation, die Argumentation oder die Konstruktionsbeschreibung erfordert. ${ }^{37}$ Dafür können im Kontext einer Lehrveranstaltung zum Beispiel, wie dies auch für den Unterricht vorgeschlagen wird, Modelltexte herangezogen oder von den Studierenden selbst erstellt werden. Eine solche fachtextsortenspezifische Diagnosefähigkeit bildet die Voraussetzung dafür, dass die Studierenden den Lernenden eine fachlich begründete Rückmeldung geben und sie zielgerichtet beim Aufbau fachlich adäquater Kommunikationsfähigkeiten unterstützen können.

\subsubsection{Hochschuldidaktische Design-Prinzipien für eine sprachbewusste Lehramtsausbildung Mathematik}

Es stellt sich nun die Frage, wie die in $\triangleright$ Abschn. 1.2.1 formulierten Leitideen in der Lehramtsausbildung Mathematik umgesetzt werden können. Dafür werden vier DesignPrinzipien (DP) vorgeschlagen - hochschuldidaktische Prinzipien also, die für die Entwicklung von Lerndesigns bzw. Lerngelegenheiten auf Hochschulebene, allgemeiner für die Entwicklung und Ausgestaltung der Hochschullehre, leitend sind:

DP1: Integration von Sprache und Fach auf struktureller und planungsbezogener Ebene in der Lehrveranstaltung

DP2: Integration von Sprache und Fach auf inhaltlicher Ebene zur Arbeit mit authentischen Lerngegenständen

DP3: Integration von Sprache und Fach auf sozialer Ebene zur Gestaltung von Arbeitsprozessen

DP4: Forschendes Lernen

Bei der Diskussion der Design-Prinzipien werden jeweils Bezüge zu relevanten Bezugsdisziplinen vorgenommen. Dazu gehören etwa allgemeine hochschuldidaktische Aspekte insbesondere zur Rolle des forschenden Lernens (in der Lehramtsausbildung), hochschuldidaktische Aspekte das Fach Mathematik betreffend sowie Einblicke in die Diskussion zur Gestaltung von Fort- und Weiterbildungsmaßnahmen im Fach Mathematik, welche ebenfalls wichtige Orientierungen zur Formulierung von Design-Prinzipien für die Hochschullehre bieten können. Auch die Hochschuldidaktik Mathematik ist als vergleichsweise junge wissenschaftliche Disziplin an der Schnittstelle von Hochschuldidaktik, Mathematikdidaktik und Mathematik ein wichtiger Bezugspunkt der vorliegenden Betrachtungen. So gibt es mittlerweile ein gut gesichertes Bild zu (eher fachbezogenen) Kompetenzen sowie der Motivationsentwicklung von Mathematikstudierenden und

37 Im Projekt SchriFT (Schreiben im Fachunterricht der Sekundarstufe I unter Einbezug des Türkischen) wurde dies im Detail für Fachtextsorten wie das Versuchsprotokoll in der Physik herausgearbeitet (Roll et al. 2019a). 
Lehramtsstudierenden mit dem Fach Mathematik (z. B. Rach 2019; Liebendörfer 2018). Ein besonderer Fokus hinsichtlich der Beforschung und Unterstützung von Studierenden (u. a. des Lehramts Mathematik) liegt dabei auf der Studieneingangsphase (z. B. Büchter et al. 2017; Kempen und Biehler 2019; Kempen 2019; Bruder et al. 2018; Grieser et al. 2018). Von zunehmender Bedeutung ist in diesem Zusammenhang auch die Beforschung von sprachlichen Aspekten im Fachstudium; so untersuchen etwa Körtling und Eichler (2019) die Entwicklung der mathematischen Sprache von Studierenden im ersten Studienjahr. Die vorliegenden Betrachtungen adressieren insbesondere die Schnittstelle von Sprache und Fach im Lehramtsstudium Mathematik, sie können daher vornehmlich an der Schnittstelle von Hochschuldidaktik, Fachdidaktik Mathematik, Sprachdidaktik, Mathematik und Sprache verortet werden.

Die Bezüge zu den jeweiligen Disziplinen erfolgen exemplarisch und erheben keinen Anspruch auf Vollständigkeit; vielmehr soll ein hochschul- und fachdidaktischer Rahmen skizziert werden, aus dem sich die Entwicklung von Design-Prinzipien für eine sprachbewusste Hochschullehre im Fach ableiten lässt.

\subsubsection{DP1: Integration von Sprache und Fach auf struktureller und planungsbezogener Ebene in der Lehrveranstaltung}

॥ Sprache hatte im Seminar die ganze Zeit „,beiläufig“ oder „,nebenbei“ einen gewissen Stellenwert. Das ist ja stellvertretend dafür, wie es später auch in unserem Unterricht sein soll. (Studentin, Praxissemester 2019).

Übergeordnetes Ziel der Integration von Sprache und Fach in der Lehramtsausbildung Mathematik ist es, dass die Studierenden eine fachbezogene Sprachbewusstheit entwickeln und flexible Handlungskompetenzen für die Gestaltung von Mathematikunterricht erwerben. Dafür müssen geeignete universitäre Lerngelegenheiten geschaffen werden, die eine strukturelle Verankerung des Themenfelds Sprache/Sprachbildung/Mehrsprachigkeit im fachdidaktischen Kontext vorsehen und eine Verzahnung theoretischer und (schul-)praktischer Phasen ermöglichen (z. B. Mavruk et al. 2017; Pitton und ScholtenAkoun 2013; Herberg und Reschke 2017; Benholz et al. 2017). Dabei sind Arbeitsaufträge grundsätzlich so angelegt, dass die Studierenden eine Doppelperspektive „Fach und Sprache" einnehmen - sei es bei der Konstruktion einer Lernumgebung, bei der Reflexion von Unterrichtsbeobachtungen oder bei der Erstellung einer Forschungsskizze. Eine solche Doppelperspektive stellt allerdings hohe Anforderungen an die Studierenden, so dass geeignete Qualifizierungs- und Unterstützungsmaßnahmen bereitgestellt werden müssen. Dazu gehört die Ermöglichung von Lerngelegenheiten, bei denen die Studierenden ihr fachbezogenes Sprachwissen systematisch erweitern: Alltägliche Spracherfahrungen und linguistische Grundkenntnisse werden aktiviert, differenziert und unter Bezug auf das Fach Mathematik konkretisiert. ${ }^{38}$ Fachliches Kommunizieren unter Bedingungen der Mehrsprachigkeit wird durch erfahrungs- und forschungsbezogene Übungen thematisiert, z. B. indem Konzeptualisierungen und Versprachlichungen fachlicher Inhalte in unterschiedlichen Sprachen verglichen werden ( Abschn. 1.1.3).

Ein weiteres Merkmal der sprachbewussten Hochschullehre ist die Verankerung gezielter Unterstützungsangebote in den Lehrveranstaltungen. Damit sind solche Angebote gemeint, die es Studierenden in der Auseinandersetzung mit den Lerngegenstän-

38 Vgl. Abschn. 2.1 für einen Vorschlag, wie das sprachliche Vorwissen von Studierenden im Kontext Mathematik spielerisch aktiviert werden kann. 
den ermöglichen, ebenjene Doppelperspektive bewusst und reflektiert einzunehmen. So fokussieren etwa Leitfragen die Aufmerksamkeit der Studierenden auf Teilaspekte fachlichen und sprachlichen Handelns und ermöglichen eine strukturierte und ausgewogene Bearbeitung der Arbeitsaufträge. Dies lässt sich grundsätzlich auf alle Themen in der Lehre anwenden: auf die Analyse von mathematischen Aufgabenstellungen ebenso wie auf die Auswertung von Schülerdokumenten oder die Entwicklung einer Forschungsfrage. Beispiele, an denen verschiedene Möglichkeiten der Integration von Fach und Sprache modellhaft gezeigt werden, haben eine wichtige Vorbildfunktion. Dies betrifft die Ebene der Fachdidaktiken (z. B. Beispiele für eine Ausgestaltung von Kernprozessen im Mathematikunterricht, die fach- und sprachdidaktische Konzepte integriert), die Ebene des fachdidaktischen und methodischen Handlungswissens (z. B. Beispiele für sprachbewusste Aufgabenvariationen), aber auch die Ebene der Forschungsmethodik (z. B. Beispiele für Methoden zur Erhebung fachlich und sprachlich interessanter Schülerprodukte).

Durch die strukturelle Verankerung ist das Thema Sprachbildung in den Lehrveranstaltungen immer präsent. Es zeigt sich also in der universitären Lehre - auch in den Formen der Unterstützung - eine gewisse Parallele zum schulischen Unterricht.

\subsubsection{DP2: Integration von Sprache und Fach auf inhaltlicher Ebene zur Arbeit mit authentischen Lerngegenständen}

॥ An den Texten, die die Schüler zu einem Funktionsgraphen geschrieben haben, ist mir aufgefallen, dass sie zwar Fachbegriffe zur Beschreibung des Graphen verwendet haben, also zum Beispiel „Diagramm“ und „X-Achse“, dass ihnen aber passende Wörter zur Beschreibung des linearen Wachstums wie ,je ... desto“ gefehlt haben. Da müsste man im Unterricht ansetzen. (Studentin, Praxissemester 2018).

Auch auf der Ebene der Lerngegenstände gehört die Integration von Sprache und Fach zu einem zentralen Design-Prinzip auf Hochschulebene. Das betrifft insbesondere die Auseinandersetzung mit schulischen Beispielen und Fällen, die den engen Zusammenhang von fachlichen und sprachlichen Aspekten im Lernprozess deutlich machen. Damit soll den Studierenden mittels Fallbezügen vor allem die Relevanz der universitären Lerngegenstände für das Studium und das spätere Tätigkeitsfeld Schule verdeutlicht werden. Dass die Arbeit mit authentischen Lerngegenständen wie Lernertexten und Unterrichtsmaterialien auch von Studierenden positiv eingeschätzt wird, bestätigen die Ergebnisse des Projekts „Sprachen - Bilden - Chancen“ (2018). Es wird entsprechend empfohlen, Praxisbezüge im Bereich Sprachbildung beizubehalten bzw. weiter auszubauen.

Auch wenn sich Erkenntnisse der Fort- und Weiterbildungsforschung natürlich nur bedingt auf die Arbeit mit Studierenden übertragen lassen, so liefern sie doch an dieser Stelle wichtige Bezugspunkte: So liegt der Wert von Fallbezügen insbesondere darin, dass sich behandelte Themen in die Praxis übersetzen lassen (Lipowsky und Rzejak 2012; für eine mathematikbezogene Konkretisierung von Fallbezügen in Fortbildungen Barzel und Selter 2015; Prediger 2019). Die empirischen Befunde zeigen darüber hinaus, dass eine wichtige Gelingensbedingung darin liegt, die eigenen (Praxis-)Erfahrungen der Teilnehmenden zum Ausgangspunkt der Fortbildungen zu machen (Borko 2004; Timperley et al. 2007). Viele der in Kap. 2 diskutierten Beispiele greifen diese Ansätze auf und adaptieren sie für die Hochschullehre. 
So können mündliche und schriftliche Dokumente aus dem Unterrichtskontext genutzt werden, um zu rekonstruieren, wie Schülerinnen und Schüler sich einem mathematischen Gegenstand sprachlich nähern, wie sie also beispielsweise funktionale Zusammenhänge anhand der Repräsentationsformen Term, Tabelle und Graph versprachlichen. Eine solche Auseinandersetzung kann wertvolle Einblicke in fachliche Aspekte der zugrunde liegenden Lernprozesse liefern, da die rekonstruierten Sprachmittel i. d. R. auf bestimmte inhaltliche Begriffsbildungen der Lernenden verweisen. Im Kontext funktionaler Zusammenhänge kann eine solche sprachlich-fachliche Analyse z. B. Hinweise darauf liefern, inwiefern die Lernenden eher auf dynamische Aspekte des Funktionsbegriffs (z. B. im Sinne der Kovariationsvorstellung, also der gemeinsamen Veränderung zweier Größen, Vollrath 1989; vom Hofe 2003) oder auf eher statische Aspekte des Funktionsbegriffs verweisen (z. B. im Sinne der Vorstellung von der Funktion als Ganzes). In \ Kap. 2 werden vielfältige Lerngegenstände aus dem Hochschulalltag vorgestellt, an denen sich die Integration von Sprache und Fach auf inhaltlicher Ebene praktisch erfahren lässt. Dabei werden hinsichtlich der Gestaltung von Arbeitsprozessen in der Hochschullehre üblicherweise drei Phasen unterschieden:

Fundierung - Für die gewinnbringende Arbeit mit Lernendendokumenten an der Schnittstelle von Sprache und Fach ist die theoretische Fundierung sowohl aus sprachlicher als auch aus fachlicher Perspektive eine wichtige Voraussetzung. Dies kann etwa für das obige Beispiel zum Thema „funktionale Zusammenhänge“ bedeuten, entsprechende fachdidaktische Grundlagenliteratur zu Grundvorstellungen zum Funktionsbegriff anzubieten sowie sprachdidaktische Grundlagenliteratur zur systematischen Unterstützung fachlichen Schreibens.

Analyse - Der - theoretisch fundierten - inhaltlichen und sprachlichen, schwerpunktmäßig eher rekonstruktiven Auseinandersetzung mit den Lerngegenständen (z. B. schriftlichen oder mündlichen Schülerdokumenten) sollte hinreichend Raum gegeben werden. Sie liefert nicht nur wertvolle Einsichten in den Zusammenhang von fachlichen und sprachlichen Aspekten der Lernprozesse, sondern unterstützt auch die sprachanalytischen und sprachdiagnostischen Kompetenzen der Studierenden im Fachkontext (Leitidee L4).

Aktion - Eine bewusst konstruktiv orientierte Auseinandersetzung mit den Lernendenbeispielen kann auf unterschiedliche Weisen erfolgen. Die in Kap. 2 diskutierten Beispiele umfassen etwa die Variation von Aufgaben und den Einsatz der Aufgaben in Diagnose- bzw. Fördersitzungen.

\subsubsection{DP3: Integration von Sprache und Fach auf sozialer Ebene zur Gestaltung von Arbeitsprozessen}

॥ Ich fand es hilfreich, sich mit den anderen in der Gruppe auszutauschen: dass man sich die Ideen gegenseitig vorstellt, darüber spricht und vielleicht auch Schwächen und Stärken herausarbeitet (Studentin, Praxissemester 2019).

॥ Gemeinsam Ideen zu entwickeln ist das, was mir am allermeisten geholfen hat; zu sehen und hören, was andere über mein Projekt denken (Student, Praxissemester 2019).

Bei der Gestaltung von Arbeitsprozessen in der sprachbewussten Hochschullehre spielt das Prinzip der Kooperation eine zentrale Rolle. Wie Ergebnisse aus der Fortbildungsforschung zeigen, ist die diskursive, reflektierte Auseinandersetzung in professionellen 
Lerngemeinschaften besonders wirkungsvoll bei der Veränderung von Handlungsroutinen (zusammenfassend Barzel und Selter 2015). Die hochschuldidaktische Forschung bestätigt, dass Aufgaben, in denen Studierende selbst aktiv werden, besonders verstehensfördernd und motivierend sind (im Überblick Svinicki und McKeachie 2014; BössOstendorf und Senft 2018)

Dabei wird insbesondere Partner- oder Gruppenarbeiten ein hohes Lernpotential zugeschrieben, die kognitiv anspruchsvoll sind und eine eigenständige, produktive Bearbeitung fachlicher Themen durch die Studierenden erfordern. Aufgrund der notwendigen gegenseitigen Unterstützung werden solche kooperativen Arbeitsformen auch in sozialer Hinsicht (nicht zuletzt mit Blick auf die spätere Berufstätigkeit) positiv eingeschätzt (Miller und Groccia 1997; Svinicki und McKeachie 2014). Durch die Arbeit in wechselnden Gruppenkonstellationen können jeweils unterschiedliche Erfahrungen und Kenntnisse in die gemeinsame Wissens- und Erkenntnisgewinnung einfließen. Für die Arbeit im Kontext Sprachbildung in der Lehramtsausbildung Mathematik spielen insbesondere folgende Faktoren eine Rolle:

- Fachwissen in den Bereichen Mathematik und Mathematikdidaktik

- Vorkenntnisse im Bereich Sprachbildung und Linguistik

- eigene sprachliche Sozialisation

- unterrichtspraktische Erfahrung

- Erfahrung mit empirischer Forschung bzw. forschendem Lernen

Die Heterogenität in den individuellen Voraussetzungen kann durch kooperatives Arbeiten für das universitäre Lernen und Forschen fruchtbar gemacht werden. Formen der Partner- und Gruppenarbeit bringen bei inhaltlich anspruchsvollen Arbeitsaufträgen die jeweiligen Kenntnisse und Erfahrungen der Studierenden zur Geltung und lassen sich auf ganz unterschiedliche Kontexte anwenden - auf die Arbeit an einer mathematischen Lernumgebung ebenso wie auf die Interpretation von Lernendendaten oder die systematische Weiterentwicklung einer Forschungsskizze.

\section{Beispiel 1}

Zwei Studierende (S1, S2) entwickeln gemeinsam eine mathematische Lernumgebung, in der Kriterien zur Sprachbildung im Fach berücksichtigt werden. S1 trägt mit ihren guten fachlichen und fachdidaktischen Kenntnissen dazu bei, dass die Aufgaben mathematisch gut fundiert und der vorgesehenen Lernphase angemessen sind. S2 hat sich in einem Praktikum verstärkt mit den Herausforderungen fachlichen Lernens für mehrsprachige Lernende auseinandergesetzt; er trägt zur Schärfung der Aufgabe aus sprachlicher Sicht bei und plant die Nutzung mehrsprachiger Ressourcen ein.

\section{Beispiel 2}

S3 hat die Eckdaten für ihr Projekt im Rahmen des Schulpraktikums ausgearbeitet. Die Mitstudierenden geben in der Gruppenarbeit dazu Rückmeldungen aus unterschiedlichen Perspektiven: S4 hat in ihrer Bachelorarbeit empirisch gearbeitet und bringt forschungsmethodische Anregungen ein. S5 schlägt vor, den mathematischen Themenbereich zu spezifizieren. S6 gibt einen Impuls, wie die Forschungsskizze im Hinblick auf das Thema Sprachbildung ausdifferenziert werden könnte.

Eine Form der Partnerarbeit in mehrfach wechselnden Konstellationen ist das „Speed-Dating“. Dieses Verfahren hat den Vorteil, dass in kurzer Zeit unterschiedliche 
Perspektiven eingeholt werden können ( Abschn. 2.6). Es eignet sich daher insbesondere in Phasen der Themenfindung als eine erste spontane Rückmeldung (z. B. zu Projektideen für studentische Forschungsvorhaben).

Die Gruppenarbeit mit Sprachbeobachtung kann zur Erhöhung des Sprachbewusstseins im Fachkontext beitragen. Dabei dokumentiert ein Studierender als Sprachbeobachter die Kommunikation der anderen Gruppenmitglieder in einem fachlichen Kontext und macht sie so der Diskussion zugänglich ( Abschn. 2.1). Dieses Verfahren eignet sich, um den Studierenden das eigene sprachliche Handeln bewusst zu machen; es bereitet zugleich auf die Analyse von Kommunikation in Lehr-Lern-Kontexten vor. Bei Gruppen mehrsprachiger Studierender mit gleichen Sprachenkonstellationen können ggf. Formen der bilingualen Kommunikation beobachtet und mit Blick auf den Unterricht thematisiert werden.

Kooperative Arbeitsprozesse müssen grundsätzlich gut vorbereitet und ggf. durch Leitfragen oder andere Maßnahmen strukturiert werden ( $\rightarrow$ DP 1). Eine (punktuelle) Unterstützung der Gruppenarbeiten durch die Lehrenden wird von den Studierenden gerade in Kontexten des forschenden Lernens als sehr hilfreich angesehen.

\subsubsection{DP4: Forschendes Lernen}

Das forschende Lernen ist gerade in der und für die Lehramtsausbildung ein zentrales Paradigma (z. B. Obolenski und Meyer 2006; Fichten 2009; Schneider 2010; Bolland 2011; Drinck 2013). Die leitende Idee besteht darin, den eigenen - universitären - Lernprozess mit forschungsbezogenen Aktivitäten zu verbinden. Breit diskutiert und implementiert wird das forschende Lernen insbesondere deswegen, weil sich Aspekte der Unterrichtsbeobachtung und -weiterentwicklung miteinander verbinden lassen und weil sich konstruktive (z. B. Entwicklung von - kleineren - Lernumgebungen) und rekonstruktive (z. B. Analyse der Herangehensweisen von Lernenden) Tätigkeiten ergänzen. Beispiele für solche Projekte werden z. B. in Abschn. 2.5 vorgestellt und diskutiert.

Theorie und Praxis werden auf diese Weise nicht nur miteinander verknüpft, sondern systematisch aufeinander bezogen: Aus forschungsbezogenen bzw. theoretischen Fundierungen ergeben sich Ansätze für kleinere empirische Projekte, die ihrerseits einen Beitrag zum Forschungsdiskurs darstellen und auch den individuellen Lernprozess wesentlich prägen können.

Vor allem bei der Planung und Durchführung von Praxisphasen im Rahmen der Lehramtsausbildung (z. B. Praxissemester, Schulpraktika etc.) spielt das forschende Lernen im Hochschulalltag eine wichtige Rolle. Auch viele der Ansätze und Beispiele dieses Buches orientieren sich am forschenden Lernen (vgl. Abschn. 1.3 und 2.5). Eine wichtige Rolle spielt dabei die Fähigkeit zum Perspektivwechsel, v. a. auch mit Blick auf die Situation von lebensweltlich mehrsprachig aufwachsenden Schüler*innen. Studierende müssen gerade im Rahmen der Fachausbildung - dafür sensibilisiert werden, wo beispielsweise neu zugewanderte Schülerinnen und Schüler zum einen fachlich und zum anderen sprachlich stehen, inwiefern Sprache im Fach im Rahmen der konkreten Beschulung adressiert wird und welche konzeptuellen Unterschiede es bei den Schulen (z. B. in der Region) hinsichtlich des Umgangs mit DaZ-Lernenden gibt. Schließlich können sich daraus erste wichtige Einsichten ergeben, die die angemessene Berücksichtigung aller Lernenden betreffen und die in die Planung kleinerer studentischer Projekte (z. B. im Rahmen von Praxisphasen) zur Integration von Sprache und Fach vor dem Hintergrund eines spezifischen Beobachtungsschwerpunktes mit einfließen können. 


\subsubsection{Leitideen und Design-Prinzipien im Überblick}

- Leitideen zur Verortung von Sprache und Fach in der Lehramtsausbildung

Leitidee L1 - Die sprachbewusste Lehramtsausbildung Mathematik sensibilisiert die Studierenden für Sprache im Fach Mathematik und schafft so die Voraussetzung für einen reflektierten Umgang mit Sprache und Mehrsprachigkeit im Mathematikunterricht.

L1-1 - Die Studierenden eignen sich spezifisches Wissen in Bezug auf die sprachlichen Eigenschaften des Fachs Mathematik an. Sie identifizieren sprachliche Anforderungen, die für das fachliche Verstehen relevant sind.

L1-2 - Die Studierenden entwickeln eine Bewusstheit für die Bedeutung von Mehrsprachigkeit im Fach Mathematik.

Leitidee L2 - Die sprachbewusste Lehramtsausbildung Mathematik befähigt die Studierenden zur Berücksichtung von Sprache bei der Arbeit mit Aufgaben.

L2-1 - Die Studierenden bestimmen und überprüfen die sprachlichen Auswirkungen mathematikdidaktisch begründeter Entscheidungen, wenn sie Aufgaben konstruieren und variieren.

L2-2 - Die Studierenden variieren Aufgaben so, dass sowohl mathematische als auch sprachliche Lernprozesse unterstützt werden.

L2-3 - Die Studierenden entwickeln Schreibaufgaben, die das mathematische Lernen unterstützen.

Leitidee L3 - Die sprachbewusste Lehramtsausbildung Mathematik befähigt die Studierenden, die Schüler*innen beim fachlichen Lernen sowie beim Ausbau von mathematisch relevanten Kommunikationsfähigkeiten systematisch zu unterstützen.

L3-1 - Die Studierenden berücksichtigen das Thema Sprache bei der Planung von Mathematikunterricht.

L3-2 - Die Studierenden entwickeln mathematische Lernumgebungen, die die Mehrsprachigkeit der Lernenden explizit adressieren.

L3-3 - Die Studierenden lernen Möglichkeiten kennen, wie sie Lernende in mündlichen Lehr-Lern-Kontexten spontan in mathematischer und sprachlicher Hinsicht unterstützen können.

Leitidee L4 - Die sprachbewusste Lehramtsausbildung Mathematik befähigt die Studierenden, bei der Diagnose von Lernendenbeiträgen fachliche und sprachliche Aspekte aufeinander zu beziehen und entsprechende Konsequenzen für das unterrichtliche Handeln im Sinne eines sprachbildenden Fachunterrichts abzuleiten.

L4-1 - Die Studierenden nutzen mündliche und schriftliche Lernendenbeiträge zur Diagnose des mathematischen Wissens und Könnens und leiten daraus entsprechende fachdidaktisch, fachlich und sprachlich begründete Fördermaßnahmen ab.

L4-2 - Die Studierenden analysieren mündliche und schriftliche Lernendenbeiträge und leiten daraus ab, über welche mathematisch relevanten sprachlichen Fähigkeiten die Lernenden schon bzw. noch nicht verfügen. Sie bestimmen den fach- und bildungssprachlichen Unterstützungsbedarf. 
- Hochschuldidaktische Design-Prinzipien für eine sprachbewusste Lehramtsausbildung Mathematik

DP1 - Integration von Sprache und Fach auf struktureller und planungsbezogener Ebene in der Lehrveranstaltung

DP2 - Integration von Sprache und Fach auf inhaltlicher Ebene zur Arbeit mit authentischen Lerngegenständen

DP3 - Integration von Sprache und Fach auf sozialer Ebene zur Gestaltung von Arbeitsprozessen

DP4 - Forschendes Lernen

\subsection{Lehramtsausbildung interdisziplinär}

I) Leistungen in Deutsch für Schülerinnen und Schüler mit Zuwanderungsgeschichte sind für alle Lehrämter zu erbringen (LABG NRW 2009, § 11, 8).

Nordrhein-Westfalen gehörte 2009 zu den ersten deutschen Bundesländern, die den Erwerb von Kenntnissen im Bereich Deutsch als Zweitsprache für alle Lehramtsstudierenden verpflichtend eingeführt haben. Das sog. DaZ-Modul, das je nach Hochschule im Bachelor und/oder im Master absolviert wird und mindestens 6 Leistungspunkte umfasst, vermittelt hierbei wichtige Grundlagenkenntnisse ( Abschn. 1.1). Um das Angebot um Veranstaltungen zu erweitern, die einen spezifischen Fachbezug herstellen, wurden z. B. an der Universität Duisburg-Essen im Rahmen des Projekts „,Deutsch als Zweitsprache in allen Fächern“ (ProDaZ) Kooperationen mit zahlreichen Fachdidaktiken, darunter Mathematik, Physik, Informatik, Chemie, Sport und Geschichte, etabliert (vgl. Abschn. 3.3 und 3.4). Zentrales Anliegen solcher Kooperationen ist es, die jeweiligen Akteure der sprachlichen und fachlichen Domänen zusammenzubringen und im Rahmen gemeinsamer Lehr- und Forschungskooperationen das Themenfeld „Sprache im Fach“ weiter zu konkretisieren. In Abschn. 3.5 werden Anregungen dafür gegeben, wie eine interdisziplinäre Kooperation aufgebaut werden kann.

Im Folgenden stellen wir exemplarisch Struktur und Inhalte der interdisziplinären Veranstaltungen für Studierende im Lehramt Mathematik vor, die im Rahmen unserer Zusammenarbeit seit 2016 entstanden sind. In den $>$ Abschn. 3.3 und 3.4 werden Beispiele für interdisziplinäre Formate in der Lehramtsausbildung Informatik und Chemie gegeben.

\subsubsection{Curriculare Verortung}

Universitäre Kooperationsseminare zum Schwerpunkt Sprachbildung im Fach sind in unterschiedlichen Phasen der Lehramtsausbildung durchführbar. Besonders fruchtbar sind solche gemeinsamen Veranstaltungen im Umfeld von Praxisphasen, weil sich vielfältige Beobachtungsanlässe und Möglichkeiten für forschendes Lernen bieten. So adressieren die hier vorgestellten Kooperationsveranstaltungen schwerpunktmäßig das Praxissemester. Dass gerade hier grundsätzlicher Handlungsbedarf besteht, zeigen Ergebnisse einer 


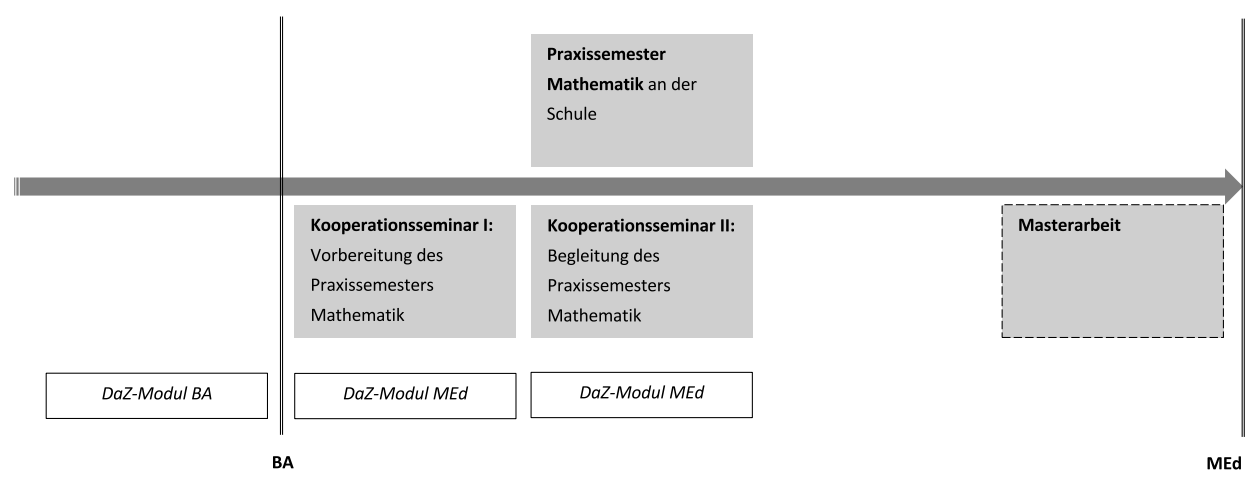

- Abb. 1.6 Curriculare Einbindung der Kooperationsseminare

entsprechenden NRW-weiten Studierendenbefragung von 2016: Im Praxissemester werden der „Erwerb von Kompetenzen in der pädagogischen Diagnostik (44\%) und die Verbindung von theoretischem Wissen und der praktischen Erfahrung (52\%)“ nicht deutlich wahrgenommen (MSW NRW 2016, S. 8); die Studierenden messen dem forschenden Lernen eine vergleichsweise geringe Relevanz bei (MSW NRW 2016, S. 8), und Studienprojekte werden als „größter Belastungsfaktor“ empfunden (MSW NRW 2016, S. 10). Die von allen beteiligten Akteuren geteilte Einschätzung ist aufgrund dieser Befundlage klar: Das forschende Lernen ,sollte daher in der Wahrnehmung der Studierenden gestützt und in der Umsetzung durch die hochschuldidaktischen Veranstaltungen in ihrer Relevanz gestärkt werden“ (MSW NRW 2016, S. 8). Dafür bieten interdisziplinäre Lehrveranstaltungen, die Sprachbildung in einem bestimmten Fach (hier: Mathematik) beleuchten, eine gute Möglichkeit, weil sie Problemstellungen aufgreifen, die für die Studierenden im Kontext des Praxissemesters unmittelbar relevant sind und im forschenden Lernen konkret untersucht werden können. Hinzu kommt, dass viele Studierende es durchaus interessant finden, ihr Fach unter dem spezifischen Aspekt der Sprachbildung neu wahrzunehmen.

Entstanden und verortet ist das Konzept im Lehramtsstudiengang Mathematik für die Schulformen Haupt-, Real-, Sekundar- und Gesamtschulen (HRSGe) im 1. und 2. Mastersemester. Es dient - wie die mathematikdidaktischen Parallelveranstaltungen ohne den Schwerpunkt Sprachbildung - der Vorbereitung und Begleitung des 5-monatigen Praxissemesters (PS) Mathematik. Im 4. Mastersemester kann sich eine Masterarbeit im Themenbereich ,Sprachbildung im Fach Mathematik“ anschließen (• Abb. 1.6).

Die Studierenden bringen Grundlagenwissen in Deutsch als Zweitsprache aus dem für die Schulformen HRSGe verpflichtenden DaZ-Modul im Bachelor (DaZ-Modul BA) sowie ggf. aus weiteren, fakultativen Veranstaltungen mit. Parallel zu den Kooperationsseminaren absolvieren sie das obligatorische DaZ-Modul im Master (DaZ-Modul MEd). ${ }^{39}$

Zudem besteht speziell an der Universität Duisburg-Essen die Möglichkeit, im Rahmen der Kooperationsseminare Leistungspunkte für die Zusatzqualifikation „Sprachbil-

39 Das DaZ-Modul im Master ist verpflichtend für Studierende der Lehrämter HRSGe und Grundschule. Studierende der Schulformen GyGe und BK können optionale Angebote über das Projekt ProDaZ wahrnehmen. 
dung in mehrsprachiger Gesellschaft" (ZuS) zu erwerben. Die Zusatzqualifikation wird seit 2014 im Kontext des Projekts „ProViel - Professionalisierung für Vielfalt“ angeboten. Sie dient der Schärfung des beruflichen Profils, indem sie Lehramtsstudierenden eine vertiefte Auseinandersetzung mit Fragen der Sprachbildung, Sprachförderung, Mehrsprachigkeit und Interkulturalität ermöglicht. ${ }^{40}$

\subsubsection{Seminarkonzeption}

Den Kern der Seminarkonzeption bildet die Auseinandersetzung mit der Rolle von Sprache und Fach vor dem, im sowie im Anschluss an das Praxissemester. Im Praxissemester besteht für Studierende - zunächst unabhängig vom studierten Lehramt - die Möglichkeit, die Beziehung von Sprache und Fach zu untersuchen, unterrichtspraktisch wirksam werden zu lassen und analytisch mit Blick auf zugrunde liegende theoretische Konzeptionen sowie die eigene Professionalität zu reflektieren. Die Beispiele und Diskussionen konzentrieren sich ganz wesentlich auf Inhalte der Vorbereitungs- und Begleitseminare zum Praxissemester Mathematik für das Lehramt HRSGe. Diese Inhalte wurden so ergänzt bzw. variiert, dass das Thema Sprachbildung sich wie ein roter Faden durch die Veranstaltungen zieht.

\section{- Das Praxissemester vorbereiten}

Die Veranstaltung Konstruktion von Lernumgebungen (KvL) im 1. Mastersemester bereitet auf das Praxissemester vor. Thematisch lassen sich solche Vorbereitungsseminare natürlich vielfältig gestalten. Im vorliegenden Beispiel ist das Seminar so ausgerichtet, dass am Beispiel des Inhaltsbereiches „Daten und Zufall“ unterschiedliche Aspekte mathematischer Lernumgebungen unter besonderer Berücksichtigung von Sprachbildung thematisiert werden. Dazu gehört sowohl die Entwicklung von Aufgaben zur Vertiefung prozessbezogener Kompetenzen (z. B. Modellieren, Argumentieren oder Begriffsbildung) als auch die Gestaltung unterschiedlicher Prozesse im Mathematikunterricht (Erkundungen, Systematisierung und Übung). Der Umgang mit mathematischen Texten im zugrundeliegenden Inhaltsbereich wird dabei ebenso thematisiert wie die Rolle von und der flexible Wechsel zwischen unterschiedlichen Repräsentationsformen.

Den Abschluss des Vorbereitungsseminars bildet die theoretisch fundierte Entwicklung einer mathematischen Lernumgebung unter Berücksichtigung von Sprachbildung (Portfolio).

Zentrale Seminarinhalte sind unter anderem:

- Aspekte zeitgemäßen und sprachbewussten Mathematikunterrichts

- Untersuchung des eigenen, evtl. mehrsprachigen, Sprachgebrauchs in mathematischen Zusammenhängen,

- Analyse und Variation von Mathematikaufgaben anhand von mathematik- und sprachdidaktischen Kriterien,

- Analyse von Kommunikation im Mathematikunterricht anhand von Transkripten und Unterrichtsbeobachtung,

- Rekonstruktion mathematischer Grund- und Fehlvorstellungen durch die Analyse von mündlichen und schriftlichen Lernerbeispielen.

40 Für Struktur und Inhalte der Zusatzqualifikation siehe $>$ https://www.uni-due.de/daz-daf/zus/. 


\section{- Das Praxissemester begleiten}

Im Mittelpunkt des Begleitseminars zum Praxissemester (2. Mastersemester) steht die Reflexion der Erfahrungen an den Schulen sowie die Planung und Durchführung eines Studienprojekts, das dem Paradigma des forschenden Lernens folgt und einen Ausschnitt aus dem Themenbereich „Sprachbildung im Mathematikunterricht“ aufgreift. Den formalen Abschluss bildet eine benotete mündliche Prüfung.

Zentrale Inhalte des Begleitseminars zum Praxissemester sind:

- Reflexion von schulischen Lehr-Lern-Prozessen unter Bezug auf die im Vorbereitungsseminar thematisierten Aspekte der Sprachbildung im Fach,

- Entwicklung von Forschungsfragen,

- Design, Durchführung und Auswertung des Studienprojekts,

- Reflexion des Forschungsprozesses im Hinblick auf die eigene Professionalisierung.

Durch die kontinuierliche Mitberücksichtigung des Themas Sprachbildung im Rahmen der Lehrveranstaltung werden die Studierenden dabei unterstützt, Fachunterricht zugleich aus fachlicher wie auch aus sprachlicher Perspektive zu betrachten und konkrete Handlungsmöglichkeiten für die Planung und Durchführung von Unterricht abzuleiten. Viele der hier thematisierten Inhalte eignen sich grundsätzlich auch für Fortbildungsveranstaltungen bzw. für die Initiierung von Lehrerhandlungs-/Aktionsforschung (Altrichter et al. 2018; Brown und Flood 2018); allerdings sind dann (u. a. aus Zeitgründen) Anpassungen notwendig.

Besonders deutlich wird die Verknüpfung von Sprache und Fach beim forschenden Lernen im Praxissemester. Aus der (durch uns gesetzten) Notwendigkeit heraus, das Thema Sprachbildung im Studienprojekt aufzugreifen, entwickeln die Studierenden oft sehr interessante Fragestellungen. Einige Beispiele:

- „Inwiefern weichen die Mathematikleistungen von Achtklässlern bei der Lösung von Textaufgaben voneinander ab, wenn die sprachlichen Anforderungen größer und kleiner werden?" (Studienprojekt 2018)

- „Wie unterscheidet sich bei mehrsprachigen Schüler*innen die Bearbeitung von Textaufgaben in der Erst- und Zweitsprache?" (Studienprojekt 2018)

- „Wie erklären Schüler*innen der Jgst. 8 die mathematischen Fachbegriffe Wahrscheinlichkeit und Zufall?“ (Studienprojekt 2019)

Dass viele Studierende die Durchführung solcher empirischen Forschungen als sehr gewinnbringend ansehen, zeigen Rückmeldungen ehemaliger Studierender. Dabei werden ganz unterschiedliche Aspekte angesprochen:

(1) Als abstrakt wahrgenommene Themen gewinnen in der Zusammenarbeit mit Schüler*innen an Anschaulichkeit. So kommentierte ein (selbst bilingual aufgewachsener) Studierender in Bezug auf das Thema „Sprache im Mathematikunterricht“: „Aus der Literatur wusste ich natürlich, dass es sprachliche Hürden gibt. Aber erst als ich im Rahmen des Studienprojekts den Kontakt zu den Schülerinnen und Schülern hatte und mit ihnen darüber geredet habe und ihre Sichtweise reflektiert habe, ist mir das Problem so richtig bewusst geworden." ( Abschn. 3.1)

(2) Die Sensibilität für Aufgabenqualität erhöht sich, da die Studierenden für ihre Forschungen selbst mathematische Lernumgebungen konzipieren, hinsichtlich ihrer fachlichen und sprachlichen Anforderungen kritisch diskutieren, überarbeiten und erproben. Damit wächst die Kritikfähigkeit in Bezug auf Aufgaben, aber auch die Kompetenz zur sprachbewussten Aufgabenkonstruktion und -variation. 
(3) Studierende nehmen die Forschungsmethoden, die sie im Rahmen ihrer Projekte kennenlernen, als Verfahren wahr, die zur Erklärung und Lösung fachlicher und/oder sprachlicher Schwierigkeiten beitragen und auch im späteren Unterricht eingesetzt werden können. Das forschende Lernen gewinnt so an handlungspraktischer Bedeutung für die zukünftige Unterrichtstätigkeit und trägt maßgeblich zur Professionalisierung der Studierenden bei.

Open Access Dieses Kapitel wird unter der Creative Commons Namensnennung 4.0 International Lizenz (http://creativecommons.org/licenses/by/4.0/deed.de) veröffentlicht, welche die Nutzung, Vervielfältigung, Bearbeitung, Verbreitung und Wiedergabe in jeglichem Medium und Format erlaubt, sofern Sie den/die ursprünglichen Autor(en) und die Quelle ordnungsgemäß nennen, einen Link zur Creative Commons Lizenz beifügen und angeben, ob Änderungen vorgenommen wurden.

Die in diesem Kapitel enthaltenen Bilder und sonstiges Drittmaterial unterliegen ebenfalls der genannten Creative Commons Lizenz, sofern sich aus der Abbildungslegende nichts anderes ergibt. Sofern das betreffende Material nicht unter der genannten Creative Commons Lizenz steht und die betreffende Handlung nicht nach gesetzlichen Vorschriften erlaubt ist, ist für die oben aufgeführten Weiterverwendungen des Materials die Einwilligung des jeweiligen Rechteinhabers einzuholen.

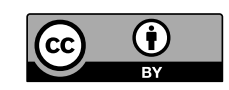

